\title{
Precise Positioning in Alpine Areas With Troposphere and Multipath Estimation
}

\author{
Patrick Henkel ${ }^{\circledR}$ and Bigyan Banjara
}

\begin{abstract}
Real-time kinematic (RTK) positioning with Global Navigation Satellite System (GNSS) signals is widely used, e.g., for surveying, agriculture, and potentially for autonomous vehicles and unmanned air vehicles in the future. In this paper, an extended RTK positioning for significant height differences between two low-cost GNSS receivers with patch antennas is presented. In this case, the classical model for double difference measurements needs to be extended, i.e., a differential tropospheric zenith delay and pseudorange multipath errors need to be estimated besides the baseline and carrier phase integer ambiguities. The increased number of unknowns results in an ill-conditioned system of observation equations and a substantial correlation between the state estimates. We introduce prior information on the differential tropospheric zenith delay based on the differential air pressure and exploit the temporal correlation of pseudorange multipath errors. Moreover, a criterion for the integer ambiguity candidate vector selection is provided, which is robust over phase multipath and unavoidable errors in the float ambiguity covariance matrix. The proposed method is validated with two low-cost GNSS modules that were placed at the Zugspitzplatt (2601 $\mathrm{m}$ a.s.l.) and Eibsee (1018 $\mathrm{m}$ a.s.l.). The obtained estimates for the baseline and differential tropospheric zenith delay show a high repeatability over several independent ambiguity fixings. The residuals of the fixed carrier phase measurements are within $2 \mathrm{~cm}$ for almost all satellites, which confirms both the measurement model and the ambiguity fixing.
\end{abstract}

Index Terms-Global navigation satellite system, meteorology, geodesy, real-time kinematics (RTK).

\section{INTRODUCTION}

$\mathbf{R}$ EAL-TIME Kinematic (RTK) positioning with GNSS carrier phase and pseudorange measurements from a kinematic receiver and a static reference station enables millimeter- to centimeter-level positioning accuracy. It is sufficient to estimate the relative position and the integer ambiguities of the periodic carrier phase measurements if the distance between both receivers is within a few kilometers and if the height difference is only in the order of a few meters. For all other cases (i.e. longer baselines or short baselines with significant height differences), the differential tropospheric delays need to be estimated and corrected. Moreover, multipath errors due to the superposition of the direct and reflected signals need

Manuscript received June 5, 2018; accepted August 5, 2018. Date of publication August 16, 2018; date of current version September 25, 2018. The associate editor coordinating the review of this paper and approving it for publication was Dr. Amitava Chatterjee. (Corresponding author: Patrick Henkel.)

The authors are with the Institute for Communications and Navigation and the Institute of Astronomical and Physical Geodesy, Technical University of Munich, 80333 Munich, Germany, and also with ANavS GmbH, 80798 Munich, Germany (e-mail: patrick.henkel@tum.de; bigyan.banjara@tum.de).

Digital Object Identifier 10.1109/JSEN.2018.2865769 to be considered for reliable RTK positioning with low-cost patch antennas.

Sahmoudi and Landry [1] proposed a particle Kalman filter for the estimation of the user's position, the carrier phase integer ambiguities and ionospheric delays. A particle filter was chosen as it is efficient for non-Gaussian states and nonlinear measurement models.

Takasu and Yasuda [2] used an extended Kalman filter for long-baseline RTK positioning with dual frequency measurements. The 3D position, ionospheric and tropospheric delays, and carrier phase ambiguities are jointly estimated in the Kalman filter.

This long-baseline set-up includes to some extent the shortbaseline set-up with a significant height difference of both receivers of this paper, i.e. the differential tropospheric zenith delays are estimated in both set-ups. However, there are two major differences: First, the ionospheric slant delays do not need to be estimated in the short-baseline set-up since the ionospheric delays are eliminated through double differencing. Secondly, the pseudorange multipath parameters need to be estimated in the considered short-baseline set-up as low-cost patch antennas are used instead of geodetic antennas and as Alpine areas are more affected by multipath.

The reliability of RTK positioning is improved by cooperative positioning of multiple receivers and/or by the integration of other sensors. Wymeersch et al. [3] provide an overview of cooperative localization techniques and applied them to UltraWide Bandwidth (UWB) wireless networks. UWB networks are attractive for short- to medium-range localization especially in GNSS-denied environments.

Georges et al. [4] proposed a sigma point belief propagation algorithm for the sensor fusion of pseudorange measurements from GNSS and Ultra-Wide Band (UWB) signals. The algorithm was designed for localization of vehicles in urban areas with Non-line-of-sight (NLOS) conditions and non-Gaussian distributed noise.

Alam and Dempster [5] analyzed the performance of cooperative positioning of vehicles with vehicular ad-hoc networks (VANETs) and radio-ranging. Shao et al. [6] and Henkel [7] analyzed the tight coupling of a low-cost GNSS receiver and a micro-electro-mechanical system (MEMS) based inertial sensor for RTK positioning and precise point positioning.

The tropospheric wet delay of the GNSS signal provides an information on the integrated water vapor. Networks of GNSS reference stations can be used to determine the horizontal distribution of the integrated water vapor with a high temporal resolution, which is very valuable for weather forecasts. Elgered et al. [8] and Bevis et al. [9] compared tropospheric 
wet zenith delay estimates from VLBI, GPS and water vapor radiometers, and obtained an accuracy between 5 and $20 \mathrm{~mm}$ for the GPS and VLBI estimates. Ahn et al. [10] estimated tropospheric corrections for RTK positioning over a short baseline during the huge typhoon RUSA, which passed over the South Korean peninsular in 2002. The RMS error of the baseline height estimate was reduced by $\sim 50 \%$ by the tropospheric corrections.

The performance of RTK positioning is improved by Virtual Reference Stations (VRS) as described by Landau et al. [11]. These VRS are located close to the receiver's position and their respective corrections are obtained by linear least-squares adjustment of the RTK corrections from the surrounding reference stations. The heart of any RTK positioning technique is the integer ambiguity fixing, e.g. by the Least-Squares AMBiguity Decorrelation Adjustment (LAMBDA) method of Teunissen [12]. It includes an integer decorrelation and a sequential tree search to find the integer ambiguities that minimize the sum of squared ambiguity residuals. The use of multiple GNSS constellations is attractive to increase the success rate or to shorten the fixing time. GLONASS is using a Frequency Division Multiple Access (FDMA) scheme, i.e. each satellite is transmitting on an individual frequency. Therefore, the double difference ambiguities are no longer integer-valued. Henkel et al. derived a transformation for GLONASS double difference ambiguities in [13] to recover the integer property.

All previous work on RTK positioning had several limitations: First, pseudorange multipath errors are widely neglected. This might be acceptable for geodetic choke-ring antennas. However, pseudorange multipath can easily reach $5 \mathrm{~m}$ for low-cost GNSS receivers and patch antennas even in environments with good satellite visibility. Secondly, RTK positioning between two GNSS receivers with a significant height difference requires an information on the differential tropospheric zenith delay. The joint estimation of baseline, integer ambiguities, differential tropospheric zenith delay and pseudorange multipath errors results in a much more ill-conditioned and challenging problem than the traditional estimation of baseline coordinates and ambiguities. Third, there is a strong need for an ambiguity fixing technique, that is robust with respect to phase multipath and unavoidable errors in the float ambiguity covariance matrix.

In this paper, we overcome the previous short-comings by an extended RTK positioning with a joint estimation of the relative position, the differential tropospheric zenith delay, the carrier phase ambiguities and the pseudorange multipath errors using GNSS measurements from multiple epochs. An integer candidate selection strategy is proposed, that is robust with respect to phase multipath, azimuthal inhomogeneities of the troposphere and unavoidable errors of the float ambiguity covariance matrix. The proposed method is tested with two low-cost GNSS receivers/ antennas being placed at the highest mountain of Germany (Zugspitze), and the surrounding valley.

This paper is organized as follows: In Section II, the models for double differenced pseudorange and carrier phase measurements and tropospheric prior information are provided.

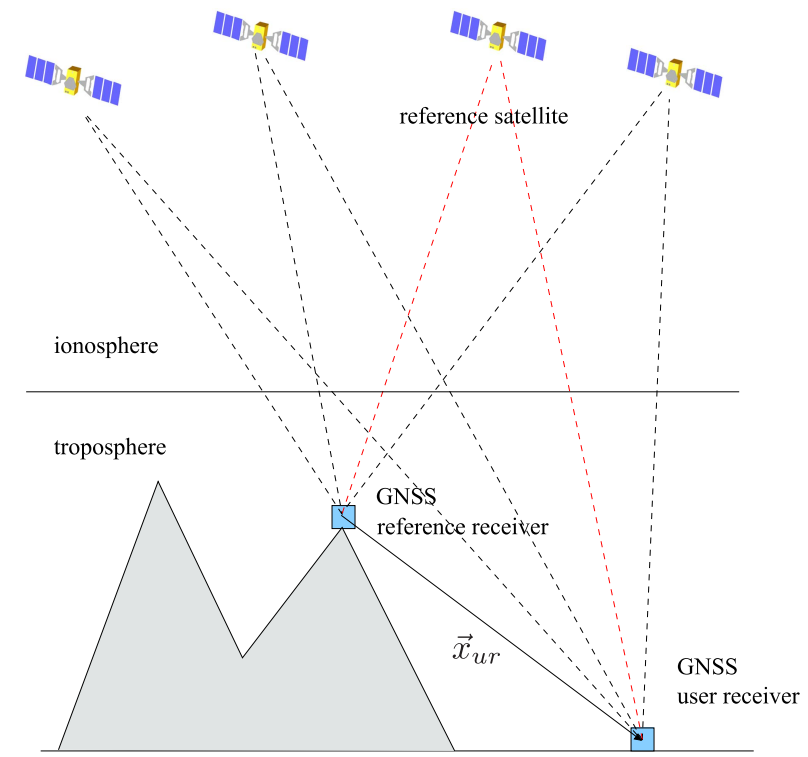

Fig. 1. Differential positioning of two GNSS receivers with significant height difference.

Section III includes the RTK positioning with troposphere and multipath estimation. A Kalman filter with integrated ambiguity fixing is used to estimate the state vector. Section IV includes a detailed description of the measurement results.

\section{Measurement Model}

In this section, we introduce the model for the differential GNSS pseudorange and carrier phase measurements.

Fig. 1 shows the assumed set-up, i.e. a GNSS user receiver is placed in a valley and a GNSS reference receiver is placed on top of a mountain. The relative position between both receivers is denoted by the baseline vector $\vec{x}_{u r}$ and determined from the differential pseudorange and carrier phase measurements. The differential tropospheric delay is not negligible due to the significant height difference between both GNSS receivers. A satellite with high elevation at both GNSS receivers is chosen as reference satellite.

A range estimate between receiver $u$ and satellite $k$ is obtained from the time of signal reception $t_{u}$ (measured by the user's clock) and the time of signal transmission $t^{k}$ (measured by the satellite's clock) and is given by

$$
r_{u}^{k}=c\left(t_{u}-t^{k}\right),
$$

with $c$ being the speed of light. The range estimation is affected by several errors including the receiver's clock error $\delta \tau_{u}$, the satellite's clock error $\delta \tau^{k}$, the tropospheric delay $T_{u}^{k}$ and ionospheric delay $I_{u}^{k}$ due to a reduced speed of signal propagation in the atmosphere, the multipath error $\Delta \varphi_{\mathrm{MP}, u}^{k}$ due to the superposition of the direct and reflected signals, the receiver and satellite biases $\beta_{u}$ and $\beta^{k}$ due to delays in the receiver- and satellite-hardware, and measurement noise $\varepsilon_{\lambda \varphi_{u}^{k}}$.

The latter term includes only by pure thermal noise and is modeled by white Gaussian noise, i.e. $\varepsilon_{\lambda \varphi_{u}^{k}} \sim \mathcal{N}\left(0, \sigma_{\varepsilon_{\lambda \varphi_{u}^{k}}^{2}}^{2}\right)$.

Range estimates can be determined from both the code and phase signals. The obtained pseudoranges and carrier 
phases are continuously tracked by the Delay Locked Loop (DLL) and Phase Locked Loop (PLL) as described by Misra and Enge [14]. The sinusoidal carrier signal is periodic with wavelength $\lambda$ and can be measured only with an ambiguity $\lambda N_{u}^{k}$ with $N_{u}^{k} \in \mathbb{Z}$. We include the previously introduced clock errors, atmospheric delays, ambiguities, biases and measurement errors in our model for the range estimate of Eq. (1), and obtain the following model for the carrier phase based range measurement (see also [14]):

$$
\begin{aligned}
\lambda \varphi_{u}^{k}= & c\left(t_{u}-t^{k}\right) \\
= & \left\|\vec{x}_{u}-\vec{x}^{k}\right\|+c\left(\delta \tau_{u}-\delta \tau^{k}\right) \\
& +T_{u}^{k}-I_{u}^{k}+\lambda \Delta \varphi_{\mathrm{MP}, u}^{k} \\
& +\lambda N_{u}^{k}+\beta_{u}+\beta^{k}+\varepsilon_{\lambda \varphi_{u}^{k}},
\end{aligned}
$$

with $\vec{x}_{u}$ and $\vec{x}^{k}$ being the positions of the $u$-th receiver and $k$-th satellite.

Differential carrier phase measurements are obtained by differencing the carrier phases of receivers $u$ and $r$. We assume a short baseline of a few kilometers with significant height difference between both GNSS receivers, i.e. the differential ionospheric delay is negligible but the differential tropospheric delay is not negligible. Thus, the single difference phase measurements are obtained from Eq. (2) and are given by

$$
\begin{aligned}
\lambda \varphi_{u r}^{k}= & \lambda \varphi_{u}^{k}-\lambda \varphi_{r}^{k} \\
= & \left\|\vec{x}_{u}-\vec{x}^{k}\right\|-\left\|\vec{x}_{r}-\vec{x}^{k}\right\|+c \delta \tau_{u r} \\
& +T_{u r}^{k}+\lambda \Delta \varphi_{\mathrm{MP}, u r}^{k}+\lambda N_{u r}^{k}+\beta_{u r}+\varepsilon_{\lambda \varphi_{u r}^{k}},
\end{aligned}
$$

where the lower index $u r$ denotes the receiver difference of the respective parameters. The receiver difference eliminates the satellite clock errors and biases. Moreover, the receiver clock errors and biases are eliminated by differencing the single difference phases $\lambda \varphi_{u r}^{k}$ and $\lambda \varphi_{u r}^{l}$ of satellites $k$ and $l$, i.e.

$$
\begin{aligned}
\lambda \varphi_{u r}^{k l}= & \lambda \varphi_{u r}^{k}-\lambda \varphi_{u r}^{l} \\
= & \left\|\vec{x}_{u}-\vec{x}^{k}\right\|-\left\|\vec{x}_{r}-\vec{x}^{k}\right\| \\
& -\left(\left\|\vec{x}_{u}-\vec{x}^{l}\right\|-\left\|\vec{x}_{r}-\vec{x}^{l}\right\|\right) \\
& +T_{u r}^{k l}+\lambda N_{u r}^{k l}+\lambda \Delta \varphi_{\mathrm{MP}, u r}^{k l}+\varepsilon_{\lambda \varphi_{u r}^{k l}} .
\end{aligned}
$$

The true receiver-satellite range is a non-linear function of the receiver's and satellite's position. The range is linearized with the normalized satellite-receiver direction vector $\vec{e}_{u}^{k}$ as

$$
\left\|\vec{x}_{u}-\vec{x}^{k}\right\|=\left(\vec{e}_{u}^{k}\right)^{\mathrm{T}}\left(\vec{x}_{u}-\vec{x}^{k}\right) \quad \forall u, k .
$$

The double difference (DD) pseudorange measurements are modeled similar to the DD carrier phases of Eq. (4), i.e.

$$
\begin{aligned}
\rho_{u r}^{k l}= & \left(\vec{e}_{u}^{k}\right)^{\mathrm{T}}\left(\vec{x}_{u}-\vec{x}^{k}\right)-\left(\vec{e}_{u}^{l}\right)^{\mathrm{T}}\left(\vec{x}_{u}-\vec{x}^{l}\right) \\
& -\left(\left(\vec{e}_{r}^{k}\right)^{\mathrm{T}}\left(\vec{x}_{r}-\vec{x}^{k}\right)-\left(\vec{e}_{r}^{l}\right)^{\mathrm{T}}\left(\vec{x}_{r}-\vec{x}^{l}\right)\right) \\
& +T_{u r}^{k l}+\Delta \rho_{\mathrm{MP}, u r}^{k l}+\varepsilon_{\rho_{u r}^{k l}},
\end{aligned}
$$

with the DD pseudorange multipath error $\Delta \rho_{\mathrm{MP}, u r}^{k l}$ and the DD pseudorange noise $\varepsilon_{\rho_{u l}^{k l}}$. The latter two parameters differ from the respective phase errors due to the different shapes of the code and phase signals.

The pseudorange noise arises only from thermal noise and is modeled by white Gaussian noise, i.e. $\varepsilon_{\rho_{u r}^{k l}} \sim \mathcal{N}\left(0, \sigma_{\varepsilon_{\rho l r}^{k l}}^{2}\right)$.
The DD carrier phase measurements of Eq. (4) are corrected for the prior knowledge of the satellite positions $\vec{x}^{k}$ and $\vec{x}^{l}$ and the reference station position $\vec{x}_{r}$, i.e.

$$
\begin{aligned}
\lambda \tilde{\varphi}_{u r}^{k l}:= & \lambda \varphi_{u r}^{k l}-\left(\left(\vec{e}_{u}^{k}\right)^{\mathrm{T}}\left(\vec{x}_{r}-\vec{x}^{k}\right)-\left(\vec{e}_{u}^{l}\right)^{\mathrm{T}}\left(\vec{x}_{r}-\vec{x}^{l}\right)\right) \\
& +\left(\left(\vec{e}_{r}^{k}\right)^{\mathrm{T}}\left(\vec{x}_{r}-\vec{x}^{k}\right)-\left(\vec{e}_{r}^{l}\right)^{\mathrm{T}}\left(\vec{x}_{r}-\vec{x}^{l}\right)\right) \\
= & \left(\vec{e}_{u}^{k}-\vec{e}_{u}^{l}\right)^{\mathrm{T}} \vec{x}_{u r}+T_{u r}^{k l}+\lambda N_{u r}^{k l} \\
& +\lambda \Delta \varphi_{\mathrm{MP}, u r}^{k l}+\varepsilon_{\lambda \varphi_{u r}^{k l}},
\end{aligned}
$$

which leaves the baseline vector $\vec{x}_{u r}=\vec{x}_{u}-\vec{x}_{r}$, the DD tropospheric delay $T_{u r}^{k l}$ and the DD integer ambiguities $N_{u r}^{k l}$ as unknowns. Similarly, the DD pseudoranges of Eq. (6) are corrected:

$$
\begin{aligned}
\tilde{\rho}_{u r}^{k l}:= & \rho_{u r}^{k l}-\left(\left(\vec{e}_{u}^{k}\right)^{\mathrm{T}}\left(\vec{x}_{r}-\vec{x}^{k}\right)-\left(\vec{e}_{u}^{l}\right)^{\mathrm{T}}\left(\vec{x}_{r}-\vec{x}^{l}\right)\right) \\
& +\left(\left(\vec{e}_{r}^{k}\right)^{\mathrm{T}}\left(\vec{x}_{r}-\vec{x}^{k}\right)-\left(\vec{e}_{r}^{l}\right)^{\mathrm{T}}\left(\vec{x}_{r}-\vec{x}^{l}\right)\right) \\
= & \left(\vec{e}_{u}^{k}-\vec{e}_{u}^{l}\right)^{\mathrm{T}} \vec{x}_{u r}+T_{u r}^{k l}+\Delta \rho_{\mathrm{MP}, u r}^{k l}+\varepsilon_{\rho_{u r}^{k l}}^{k l} .
\end{aligned}
$$

The tropospheric delay $T_{u}^{k}$ is split into a dry/ hydrostatic component and a wet component as proposed by Davis et al. [15]. A homogeneous troposphere is assumed in the surroundings of the receiver, i.e. the dry/ wet component of the tropospheric delay is modeled as the product of a zenith delay $T_{\mathrm{z}, \mathrm{dry} / \text { wet }, u}$ and a mapping function $m_{\mathrm{dry} / \text { wet }, u}^{k}$ for projecting the zenith delay into the slant direction, i.e.

$$
T_{u}^{k}=m_{\mathrm{dry}, u}^{k} T_{\mathrm{z}, \mathrm{dry}, u}+m_{\mathrm{wet}, u}^{k} T_{\mathrm{z}, \mathrm{wet}, u} .
$$

We refer to Ahn et al. [10] for the determination of tropospheric corrections during localized severe weather events with an inhomogeneous troposphere. The double difference tropospheric delay $T_{u r}^{k l}$ is obtained from Eq. (9) by

$$
\begin{aligned}
T_{u r}^{k l}= & T_{u}^{k l}-T_{r}^{k l} \\
= & m_{\mathrm{dry}, u}^{k l} T_{\mathrm{z}, \mathrm{dry}, u}-m_{\mathrm{dry}, r}^{k l} T_{\mathrm{z}, \mathrm{dry}, r} \\
& +m_{\mathrm{wet}, u}^{k l} T_{\mathrm{z}, \mathrm{wet}, u}-m_{\mathrm{wet}, r}^{k l} T_{\mathrm{z}, \mathrm{wet}, r} \\
\approx & m_{\mathrm{dry}, u}^{k l}\left(T_{\mathrm{z}, \mathrm{dry}, u}-T_{\mathrm{z}, \mathrm{dry}, r}\right) \\
& +m_{\mathrm{wet}, u}^{k l}\left(T_{\mathrm{z}, \mathrm{wet}, u}-T_{\mathrm{z}, \mathrm{wet}, r}\right) \\
\approx & m_{\mathrm{dry}, u}^{k l}\left(T_{\mathrm{z}, \mathrm{dry}, u r}+T_{\mathrm{z}, \mathrm{wet}, u r}\right),
\end{aligned}
$$

whereas we assumed equal mapping functions for receivers $u$ and $r$ (due to the small baseline) in the first approximation and equal mapping functions for the dry and wet component in the second approximation. The second approximation can be used if satellites of very low elevation $\left(<10^{\circ}\right)$ are disregarded. The differential tropospheric zenith delays $T_{z, \text { dry, } u r}$ are only a fraction of the total tropospheric delay, i.e. small errors in the mapping function are suppressed by the differential measurements.

We also consider prior information on the differential tropospheric zenith delay. It includes a hydrostatic (dry) and a wet component as described by Davis et al. [15]:

$$
\bar{T}_{\mathrm{z}, u r}=\bar{T}_{\mathrm{z}, \mathrm{dry}, u r}+\bar{T}_{\mathrm{z}, \mathrm{wet}, u r} .
$$


The differential hydrostatic part is modeled according to Elgered et al. [8] in terms of the total air pressure $p$ at the $u$-th and $r$-th receiver (in units of bars), i.e.

$\bar{T}_{\mathrm{z}, \mathrm{dry}, u r}=(2.2779 \pm 0.0024)\left(\frac{p\left(h_{u}\right)}{f\left(\phi_{u}, h_{u}\right)}-\frac{p\left(h_{r}\right)}{f\left(\phi_{r}, h_{r}\right)}\right) \frac{\mathrm{m}}{\mathrm{bar}}$,

where the function $f\left(\phi_{u}, h_{u}\right)$ models the variation of the acceleration due to gravity. It depends on the latitude $\phi_{u}$ and height $h_{u}$, and is given by:

$$
f\left(\phi_{u}, h_{u}\right)=1-0.00266 \cos \left(2 \phi_{u}\right)-0.28 \cdot 10^{-3} \cdot h_{u} / \mathrm{km} .
$$

This function is not very sensitive with respect to small changes of the position. Therefore, we assume an equal $f$ for the $u$-th and $r$-th receiver being separated by only a few kilometers. If a calibrated air pressure measurement is not available at the receiver's location, the air pressure could be modeled according to [16] as

$$
p(h)=p_{0}\left(h_{0}\right) \cdot(1-\delta(h))^{\gamma},
$$

with

$$
\delta(h)=\frac{\alpha\left(h-h_{0}\right)}{T_{0}\left(h_{0}\right)} \quad \text { and } \quad \gamma=\frac{g}{R_{\mathrm{d}} \alpha},
$$

with the height $h$, the air pressure $p_{0}$ at height $h_{0}$, the temperature lapse rate $\alpha$ (the US standard atmosphere assumes a value of $6.5 \mathrm{~K} / \mathrm{km}$ ), the temperature $T_{0}$ at height $h_{0}$, the gravitational acceleration $g=9.80 \mathrm{~m} / \mathrm{s}^{2}$ and the specific gas constant $R_{\mathrm{d}}=287.1 \frac{\mathrm{J}}{\mathrm{kg} \cdot \mathrm{K}}$ for dry air.

In Eq. (15), the ratio $\delta(h)$ is much smaller than 1 for all relevant heights (i.e. $h-h_{0}<10 \mathrm{~km}$ ). Therefore, the second factor of Eq. (14) is approximated by a Taylor series expansion up to order two, i.e.

$$
\begin{aligned}
f(\delta):= & (1-\delta)^{\gamma} \\
\approx & \left.f(\delta)\right|_{\delta=0}+\left.\frac{\partial f(\delta)}{\partial \delta}\right|_{\delta=0} \cdot \delta+\left.\frac{\partial^{2} f(\delta)}{\partial \delta^{2}}\right|_{\delta=0} \cdot \frac{\delta^{2}}{2} \\
= & 1-\left.\gamma(1-\delta)^{\gamma-1}\right|_{\delta=0} \cdot \delta \\
& +\left.\gamma(\gamma-1)(1-\delta)^{\gamma-2}\right|_{\delta=0} \cdot \frac{\delta^{2}}{2} \\
= & 1-\gamma \cdot \delta+\gamma(\gamma-1) \cdot \frac{\delta^{2}}{2}
\end{aligned}
$$

The differential air pressure follows from Eq. (14) - (16) as

$$
\begin{aligned}
p\left(h_{u}\right)-p\left(h_{r}\right) & \\
\approx p\left(h_{0}\right) \cdot( & \left.\left(f\left(h_{u}\right)\right)-f\left(\delta\left(h_{r}\right)\right)\right) \\
=p\left(h_{0}\right) & \cdot\left(-\gamma \frac{\alpha}{T_{0}\left(h_{0}\right)} \cdot\left(h_{u}-h_{r}\right)+\frac{\gamma(\gamma-1)}{2} \frac{\alpha^{2}}{T_{0}^{2}\left(h_{0}\right)}\right. \\
& \left.\cdot\left(\left(h_{u}-h_{0}\right)^{2}-\left(h_{r}-h_{0}\right)^{2}\right)\right) .
\end{aligned}
$$

The wet component of the tropospheric zenith delay varies between a few centimeters or less in arid regions and $35 \mathrm{~cm}$ in humid regions according to Bevis et al. [9]. In this paper, we consider a differential positioning with a height difference of only $\sim 1.5 \mathrm{~km}$ on a dry day, such that the wet component of the differential zenith delay is assumed to be within $2 \mathrm{~cm}$.
This small magnitude of the wet differential zenith delay and the use of an elevation mask of $10^{\circ}$ allow the use of a common mapping function for both dry and wet components.

The DD carrier phase and pseudorange measurements from all satellites of epoch $n$ and the prior information $\bar{T}_{\mathrm{z}, u r}$ on the differential tropospheric zenith delay are stacked in a single vector $z_{n}$ :

$$
z_{n}=\left(\begin{array}{c}
\lambda \tilde{\varphi}_{u r} \\
\tilde{\rho}_{u r} \\
\bar{T}_{\mathrm{z}, u r}
\end{array}\right),
$$

where $\lambda \tilde{\varphi}_{u r}$ and $\tilde{\rho}_{u r}$ denote the stacked DD carrier phase and pseudorange measurements of all satellites:

$$
\lambda \tilde{\varphi}_{u r}=\left(\lambda \tilde{\varphi}_{u r}^{1 l}, \ldots, \lambda \tilde{\varphi}_{u r}^{K l}\right)^{\mathrm{T}}, \quad \tilde{\rho}_{u r}=\left(\tilde{\rho}_{u r}^{1 l}, \ldots, \tilde{\rho}_{u r}^{K l}\right)^{\mathrm{T}},
$$

with $\lambda \tilde{\varphi}_{u r}^{k l}$ given by Eq. (7) and $\tilde{\rho}_{u r}^{k l}$ given by Eq. (8). The unknown parameters of Eq. (7)-(8) are stacked in a state vector, i.e.

$$
x_{n}=\left(\begin{array}{c}
\vec{x}_{u r} \\
T_{z, u r} \\
N_{u r} \\
\Delta \rho_{\mathrm{MP}, u r}
\end{array}\right),
$$

with the baseline vector $\vec{x}_{u r}$, the differential tropospheric zenith delay $T_{z, u r}$, the DD integer ambiguities $N_{u r}$ and the differential pseudorange multipath errors $\Delta \rho_{\mathrm{MP}, u r}$. The latter quantities are obtained by stacking the DD ambiguities and pseudorange multipath errors of all satellites, i.e.

$$
\begin{aligned}
N_{u r} & =\left(N_{u r}^{1 l}, \ldots, N_{u r}^{K l}\right)^{\mathrm{T}} \\
\Delta \rho_{\mathrm{MP}, u r} & =\left(\Delta \rho_{\mathrm{MP}, u r}^{1 l}, \ldots, \Delta \rho_{\mathrm{MP}, u r}^{K l}\right)^{\mathrm{T}} .
\end{aligned}
$$

The measurement models of Eq. (7) and (8) are used to relate the measurement vector $z_{n}$ of Eq. (18) to the state vector $x_{n}$ of Eq. (20). The obtained linear relationship is written as

$$
z_{n}=H_{n} x_{n}+\varepsilon_{z_{n}},
$$

with the mapping matrix $H_{n}$ obtained from Eq. (7)-(11) as

$$
H_{n}=\left(\begin{array}{cccccccc}
\left(\vec{e}_{u}^{1 l}\right)^{\mathrm{T}} & m_{r}^{1 l} & \lambda & & & 0 & \ldots & 0 \\
\vdots & \vdots & & \ddots & & \vdots & \ddots & \vdots \\
\left(\vec{e}_{u}^{K l}\right)^{\mathrm{T}} & m_{r}^{K l} & & & \lambda & 0 & \ldots & 0 \\
\hline\left(\vec{e}_{u}^{1 l}\right)^{\mathrm{T}} & m_{r}^{1 l} & 0 & \ldots & 0 & 1 & & 0 \\
\vdots & \vdots & \vdots & \ddots & \vdots & & \ddots & \\
\left(\vec{e}_{u}^{K l}\right)^{\mathrm{T}} & m_{r}^{K l} & 0 & \ldots & 0 & 0 & & 1 \\
\hline 0^{1 \times 3} & 1 & 0 & \ldots & 0 & 0 & \ldots & 0
\end{array}\right),
$$

and the white Gaussian noise vector

$$
\varepsilon_{z_{n}}=\left(\varepsilon_{\lambda \varphi_{u r}}^{\mathrm{T}}, \varepsilon_{\rho_{u r}}^{\mathrm{T}}, \varepsilon_{\bar{T}_{z, u r}}\right)^{\mathrm{T}} \sim \mathcal{N}\left(0, \Sigma_{z_{n}}\right),
$$

with the stacked phase noise terms $\varepsilon_{\lambda \varphi_{u r}}=$ $\left(\varepsilon_{\lambda \varphi_{u r}^{1 l}}, \ldots, \varepsilon_{\lambda \varphi_{u r}^{K l}}\right)^{\mathrm{T}}$ and pseudorange noise terms $\varepsilon_{\rho_{u r}}=\left(\varepsilon_{\rho_{u r}^{1 l}}, \ldots, \varepsilon_{\rho_{u r}^{K l}}\right)^{\mathrm{T}}$ of all satellites and the scalar error $\varepsilon_{\bar{T}_{z, u r}}$ of the tropospheric prior information. The mapping matrix $H_{n}$ of a single epoch consists of $2(K-1)+1$ 
rows and $4+2(K-1)$ columns and, therefore, is rank-defect. However, the use of measurements of multiple epochs results in an over-determined system of equations as the ambiguities are constant over time.

\section{A. Multipath Modeling}

We model the received signal at time $t$ as the superposition of the direct signal with amplitude $A_{0}$, chip form $c(t)$, code delay $\tau$, phase $\varphi$ and Doppler frequency $\omega$ and $L$ reflected signals with amplitude $A_{l}$, extra path delay $\Delta t_{l}$, phase shift $\Delta \varphi_{l}$ and Doppler offset $\Delta \omega_{l}$ according to Henkel et al. [18] as:

$$
\begin{aligned}
r(t)= & A_{0} \cdot c(t-\tau) \cdot e^{j(\varphi+\omega(t-\tau))} \\
& +\sum_{l=1}^{L} A_{l} \cdot c\left(t-\left(\tau+\Delta t_{l}\right)\right) \\
& \cdot e^{j\left(\varphi+\Delta \varphi_{l}+\left(\omega+\Delta \omega_{l}\right)\left(t-\left(\tau+\Delta t_{l}\right)\right)\right)}+\eta(t),
\end{aligned}
$$

with $\eta$ being the noise of the received signal. We assume that all tracking loops (DLL, FLL and PLL) are in lock. In this case, we can remove the carrier from the received signal by multiplying $r(t)$ with the conjugate complex of $e^{j(\varphi+\omega(t-\tau))}$, i.e.

$$
\begin{aligned}
r^{*}(t)= & r(t) \cdot e^{-j(\varphi+\omega(t-\tau))} \\
= & A_{0} \cdot c(t-\tau)+\sum_{l=1}^{L} A_{l} \cdot c\left(t-\left(\tau+\Delta t_{l}\right)\right) \\
& \cdot e^{j\left(\Delta \varphi_{l}-\omega \Delta t_{l}-\Delta \omega_{l}\left(t-\left(\tau+\Delta t_{l}\right)\right)\right)}+\eta(t) .
\end{aligned}
$$

As the signal of interest is only in the real part of $r^{*}(t)$, we can eliminate the imaginary part of the reflected signals by a projection onto the space of real-valued numbers, i.e.

$$
\begin{aligned}
\operatorname{Re}\left\{r^{*}(t)\right\} & \\
= & A_{0} \cdot c(t-\tau) \\
& +\sum_{l=1}^{L} A_{l} \cdot c\left(t-\left(\tau+\Delta t_{l}\right)\right) \\
& \cdot \cos \left(\Delta \varphi_{l}-\omega \Delta t_{l}-\Delta \omega_{l}\left(t-\left(\tau+\Delta t_{l}\right)\right)\right)+\eta(t) .
\end{aligned}
$$

The GNSS receiver correlates $\operatorname{Re}\left\{r^{*}(t)\right\}$ with the receivergenerated baseband signal $A_{0} \cdot c(t-\tau-\Delta \tau)$ and obtains as correlation result:

$$
C(\Delta \tau)=\int_{0}^{T_{\mathrm{c}}} \operatorname{Re}\left\{r^{*}(t)\right\} \cdot A_{0} \cdot c(t-\tau-\Delta \tau) d(t-\tau),
$$

with $T_{\mathrm{c}}$ being the integration period. Replacing $\operatorname{Re}\left\{r^{*}(t)\right\}$ by Eq. (27) gives

$$
\begin{aligned}
C(\Delta \tau)= & \int_{0}^{T_{\mathrm{c}}} A_{0}^{2} c(t-\tau) c(t-\tau-\Delta \tau) d(t-\tau)+\Delta C_{\mathrm{MP}}(\Delta \tau) \\
& +\int_{0}^{T_{\mathrm{c}}} \eta(t) A_{0} c(t-\tau-\Delta \tau) d(t-\tau)
\end{aligned}
$$

where the multipath-induced error $\Delta C_{\mathrm{MP}}(\Delta \tau)$ of the correlation result is given by

$$
\begin{aligned}
& \Delta C_{\mathrm{MP}}(\Delta \tau) \\
& =\sum_{l=1}^{L} \int_{0}^{T_{\mathrm{c}}} A_{0} A_{l} c\left(t-\left(\tau+\Delta \tau_{l}\right)\right) c(t-\tau-\Delta \tau) \\
& \quad \cdot \cos \left(\Delta \varphi_{l}-\omega \Delta \tau_{l}-\Delta \omega_{l}\left(t-\left(\tau+\Delta t_{l}\right)\right)\right) d(t-\tau) .
\end{aligned}
$$

The code chip $c(t)$ is modeled by a rectangular pulse shape

$$
c(t)=\left\{\begin{array}{ll}
1 & \text { if } 0 \leq t \leq T_{\mathrm{c}} \\
0 & \text { else }
\end{array},\right.
$$

which enables us to further evaluate the first integral in Eq. (29):

$$
\begin{aligned}
\int_{0}^{T_{\mathrm{c}}} A_{0}^{2} c(t-\tau) c(t & -\tau-\Delta \tau) d(t-\tau) \\
& =A_{0}^{2} \cdot \begin{cases}T_{\mathrm{c}}-|\Delta \tau| & \text { if }|\Delta \tau|<T_{\mathrm{c}} \\
0 & \text { else. }\end{cases}
\end{aligned}
$$

Discriminator functions are used to extract the code delay offset $\Delta \tau$ from the correlation result. The Early minus Late discriminator (see Misra and Enge [14]) is obtained from the difference of the early and late correlation results $C_{\mathrm{E} / \mathrm{L}}$, i.e.

$$
\begin{aligned}
D(\Delta \tau) & =C_{\mathrm{E}}(\Delta \tau)-C_{\mathrm{L}}(\Delta \tau) \\
& =C\left(\Delta \tau-\frac{d T_{\mathrm{c}}}{2}\right)-C\left(\Delta \tau+\frac{d T_{\mathrm{c}}}{2}\right),
\end{aligned}
$$

with the discriminator spacing $d$. The discriminator function $D(\Delta \tau)$ is 0 for $\Delta \tau=0$ in the absence of multipath. Otherwise, the zero-crossing of the discriminator function is biased. As the delay locked loop (DLL) controls $\Delta \tau$ to be around 0 , the effective pseudorange multipath error $\Delta \tau_{\mathrm{MP}}$ is implicitly defined by

$$
D\left(\Delta \tau_{\mathrm{MP}}\right)=\Delta C_{\mathrm{E}}\left(\Delta \tau_{\mathrm{MP}}\right)-\Delta C_{\mathrm{L}}\left(\Delta \tau_{\mathrm{MP}}\right) \stackrel{!}{=} 0 .
$$

The GNSS measurements are taken at discrete epochs. Therefore, we switch from time-continuous to time-discrete notation and model the amplitude $A_{l, n}$, extra-path delay $\Delta \tau_{l, n}$, phase shift $\Delta \varphi_{l, n}$ and Doppler offset $\Delta \omega_{l, n}$ of the $l$-th reflection at epoch $n$ by random-walk processes, i.e.

$$
\begin{aligned}
A_{l, n} & =A_{l, n-1}+\eta_{A_{l, n}} \\
\Delta \tau_{l, n} & =\Delta \tau_{l, n-1}+\eta_{\Delta \tau_{l, n}} \\
\Delta \varphi_{l, n} & =\Delta \varphi_{l, n-1}+\eta_{\Delta \varphi_{l, n}} \\
\Delta \omega_{l, n} & =\Delta \omega_{l, n-1}+\eta_{\Delta \omega_{l, n}},
\end{aligned}
$$

with $\eta_{A_{l, n}}, \eta_{\Delta \tau_{l, n}}, \eta_{\Delta \varphi_{l, n}}$ and $\eta_{\Delta \omega_{l, n}}$ describing the change of the amplitude, extra-path delay, phase shift and Doppler offset between epoch $n-1$ and $n$. The pseudorange multipath errors $\Delta \rho_{\mathrm{MP}, u r}$ follow from Eq. (30) - (34) in accordance with [18]. The time behavior of the multipath errors $\left(\Delta \rho_{\mathrm{MP}, u r}\right)_{n}=$ $c\left(\Delta \tau_{\mathrm{MP}_{u r}}\right)_{n}$ is modeled based on linearization of $\Delta \tau_{\mathrm{MP}}$ and Eq. (35) as

$$
\left(\Delta \rho_{\mathrm{MP}, u r}\right)_{n}=\left(\Delta \rho_{\mathrm{MP}, u r}\right)_{n-1}+\left(\varepsilon_{\Delta \rho_{\mathrm{MP}, u r}}\right)_{n},
$$

with $\left(\varepsilon_{\Delta \rho_{\mathrm{MP}, u r}}\right)_{n}$ being the change of the multipath error between epochs $n-1$ and $n$. 


\section{B. State Space Model}

The state vector $x_{n}$ of Eq. (20) is related to the state vector of the previous epoch by the following linear state space model:

$$
x_{n}=\Phi_{n} x_{n-1}+\eta_{x_{n}},
$$

with the state transition matrix $\Phi_{n}$ and the process noise $\eta_{x_{n}}$. The latter one is assumed to be Gaussian distributed, i.e.

$$
\eta_{x_{n}} \sim \mathcal{N}\left(0, Q_{x}\right),
$$

with $Q_{x}$ being the process noise covariance matrix. We consider a static positioning, i.e. the baseline $\vec{x}_{u r}$ is constant. The integer ambiguities are also constant if the phase locked loop is in lock and no cycle slips occur. Moreover, the tropospheric zenith delay can be assumed to be constant over short periods of time.

The code multipath errors are modeled by a random-walk process as described in Eq. (36). Thus, the state transition matrix becomes an identity matrix $I$, i.e.

$$
\Phi_{n}=I^{4+2(K-1) \times 4+2(K-1)},
$$

with $K$ being the number of satellites and $2(K-1)$ being the number of double difference ambiguity and code multipath parameters.

As the code multipath errors are the only state parameters that change with time, the process noise covariance matrix is given by

$$
Q_{x}=\left(\begin{array}{cc}
0^{(4+K-1) \times(4+K-1)} & 0^{(4+K-1) \times(K-1)} \\
0^{(K-1) \times(4+K-1)} & \Sigma_{\Delta \rho_{\mathrm{MP}}}
\end{array}\right),
$$

with $\Sigma_{\Delta \rho_{\mathrm{MP}}}$ describing the process noise of the pseudorange multipath errors.

\section{RTK POSITIONING WITH TROPOSPHERE and Multipath Estimation}

In this section, the RTK positioning with troposphere and multipath estimation is described using the measurement and state space model of the previous section.

We use a Kalman filter and derive it from belief propagation using a linear Gaussian model [22].

\section{A. Kalman Filter Based Float Solution}

The joint probability distribution of the state parameter vector $\boldsymbol{x}=\left[x_{1}^{\mathrm{T}}, \ldots, x_{n}^{\mathrm{T}}\right]^{\mathrm{T}}$ and the GPS measurement vector $z=\left[z_{1}^{\mathrm{T}}, \ldots, z_{n}^{\mathrm{T}}\right]^{\mathrm{T}}$ of all epochs $j=\{1, \ldots, n\}$ is given by

$$
p(\boldsymbol{x}, \boldsymbol{z})=p(\boldsymbol{x}) p(z \mid \boldsymbol{x})=\prod_{j=1}^{n} p\left(x_{j}\right) \prod_{j=1}^{n} p\left(z_{j} \mid x_{j}\right) .
$$

We assume that the prior information on the state parameters $x_{j}$ is Gaussian distributed, i.e.

$$
p\left(x_{j}\right)=\frac{1}{\sqrt{(2 \pi)^{n_{x}}\left|\Sigma_{\bar{x}_{j}}\right|}} e^{-\frac{1}{2}\left\|x_{j}-\bar{x}_{j}\right\|_{\Sigma_{\bar{x}_{j}}^{-1}}^{2},}
$$

with $n_{x}$ being the number of state parameters, $\bar{x}_{j}$ being the prior information on the state parameters of epoch $j$ and
$\Sigma_{\bar{x}_{j}}$ being the covariance matrix of the prior information. We additionally assume that the measurement vector conditioned on the state vector is also Gaussian distributed, i.e.

$$
p\left(z_{j} \mid x_{j}\right)=\frac{1}{\sqrt{(2 \pi)^{n_{z}\left|\Sigma_{z_{j}}\right|}}} e^{-\frac{1}{2}\left\|z_{j}-H_{j} x_{j}\right\|_{\Sigma_{z_{j}}}^{2}},
$$

with $n_{z}$ being the number of measurements, $H_{j}$ being the mapping matrix of the state parameters into the measurements, and $\Sigma_{z_{j}}$ being the measurement covariance matrix. The state parameters shall be determined such that the joint probability of Eq. (41) is maximized, i.e.

$$
\left(\begin{array}{c}
\hat{x}_{1} \\
\vdots \\
\hat{x}_{n}
\end{array}\right)=\arg \max _{x_{1}, \ldots, x_{n}}\left(\prod_{j=1}^{n} p\left(x_{j}\right) \prod_{j=1}^{n} p\left(z_{j} \mid x_{j}\right)\right)
$$

The maximization over all states can be split into a maximization over the state vector of the last epoch and a maximization over all previous states, i.e.

$$
\max _{x_{1}, \ldots, x_{n-1}}\left(\prod_{j=1}^{n-1} p\left(x_{j}\right) \prod_{j=1}^{n-1} p\left(z_{j} \mid x_{j}\right)\right) \cdot \max _{x_{n}}\left(p\left(x_{n}\right) p\left(z_{n} \mid x_{n}\right)\right) .
$$

We consider a sequential approach, i.e. it is assumed that all previous states $\left\{x_{1}, \ldots, x_{n-1}\right\}$ were determined in a previous step and that only the current state parameters need to be determined at the current step. We replace the probabilities in the second maximization of Eq. (45) by Eq. (42) and (43), and take into account that the maximization of an exponential function with negative exponent corresponds to a minimization of the absolute value of the exponent, i.e.

$$
\hat{x}_{n}^{+}=\arg \min _{x_{n}}\left(\left\|x_{n}-\bar{x}_{n}\right\|_{\Sigma_{\bar{x}_{n}}^{-1}}^{2}+\left\|z_{n}-H_{n} x_{n}\right\|_{\Sigma_{z_{n}}^{-1}}^{2}\right),
$$

where the prior information $\bar{x}_{n}$ is obtained from Eq. (37) as

$$
\bar{x}_{n}=\Phi \hat{x}_{n-1}^{+},
$$

and the respective covariance matrix is given by

$$
\Sigma_{\bar{x}_{n}}=\Phi \Sigma_{\hat{x}_{n-1}^{+}} \Phi^{\mathrm{T}}+Q_{x},
$$

with the process noise covariance matrix $Q_{x}$.

The sum of two squared norms in Eq. (46) can be rearranged into a single squared norm, i.e.

$$
\hat{x}_{n}^{+}=\arg \min _{x_{n}}\left\|\left(\begin{array}{c}
\bar{x}_{n} \\
z_{n}
\end{array}\right)-\left(\begin{array}{c}
I \\
H_{n}
\end{array}\right) x_{n}\right\|_{\Sigma^{-1}}^{2},
$$

with

$$
\Sigma=\left(\begin{array}{cc}
\Sigma_{\bar{x}_{n}} & 0 \\
0 & \Sigma_{z_{n}}
\end{array}\right) .
$$

The least-squares solution of Eq. (49) is given by

$$
\hat{x}_{n}^{+}=\left(\Sigma_{\bar{x}_{n}}^{-1}+H_{n}^{\mathrm{T}} \Sigma_{z_{n}}^{-1} H_{n}\right)^{-1}\left(\Sigma_{\bar{x}_{n}}^{-1} \bar{x}_{n}+H_{n}^{\mathrm{T}} \Sigma_{z_{n}}^{-1} z_{n}\right)
$$


We use the matrix-inversion lemma (Sherman-MorrisonWoodbury formula of [23]) to rewrite the matrix inversion of Eq. (51) as

$$
\begin{aligned}
\left(\Sigma_{\bar{x}_{n}}^{-1}+\right. & \left.H_{n}^{\mathrm{T}} \Sigma_{z_{n}}^{-1} H_{n}\right)^{-1} \\
& =\Sigma_{\bar{x}_{n}}-\Sigma_{\bar{x}_{n}} H_{n}^{\mathrm{T}}\left(\Sigma_{z_{n}}+H_{n} \Sigma_{\bar{x}_{n}} H_{n}^{\mathrm{T}}\right)^{-1} H_{n} \Sigma_{\bar{x}_{n}} .
\end{aligned}
$$

Replacing the matrix inversion of Eq. (51) by Eq. (52) and further simplifying the first term gives

$$
\begin{aligned}
\left(\Sigma_{\bar{x}_{n}}^{-1}+H_{n}^{\mathrm{T}} \Sigma_{z_{n}}^{-1} H_{n}\right)^{-1} \Sigma_{\bar{x}_{n}}^{-1} \bar{x}_{n} & \\
& =\bar{x}_{n}-K_{n} H_{n} \bar{x}_{n}=\left(1-K_{n} H_{n}\right) \bar{x}_{n},
\end{aligned}
$$

with the Kalman gain

$$
K_{n}=\Sigma_{\bar{x}_{n}} H_{n}^{\mathrm{T}}\left(\Sigma_{z_{n}}+H_{n} \Sigma_{\bar{x}_{n}} H_{n}^{\mathrm{T}}\right)^{-1}
$$

The second component of Eq. (51) is rewritten with the second matrix inversion lemma as

$$
\begin{aligned}
\left(\Sigma_{\bar{x}_{n}}^{-1}+H_{n}^{\mathrm{T}} \Sigma_{z_{n}}^{-1} H_{n}\right)^{-1} H_{n}^{\mathrm{T}} \Sigma_{z_{n}}^{-1} z_{n} \\
\quad=\Sigma_{\bar{x}_{n}} H_{n}^{\mathrm{T}}\left(\Sigma_{z_{n}}+H_{n} \Sigma_{\bar{x}_{n}} H_{n}^{\mathrm{T}}\right)^{-1} z_{n} \\
=K_{n} z_{n} .
\end{aligned}
$$

Combining Eq. (53) and (55) gives the state update

$$
\hat{x}_{n}^{+}=\left(1-K_{n} H_{n}\right) \bar{x}_{n}+K_{n} z_{n}=\bar{x}_{n}+K_{n}\left(z_{n}-H_{n} \bar{x}_{n}\right) \text {, }
$$

and its covariance matrix follows as

$$
\begin{aligned}
\Sigma_{\hat{x}_{n}^{+}} & =\left(1-K_{n} H_{n}\right) \Sigma_{\bar{x}_{n}}\left(1-K_{n} H_{n}\right)^{\mathrm{T}}+K_{n} \Sigma_{z_{n}} K_{n}^{\mathrm{T}} \\
& =\left(1-K_{n} H_{n}\right) \Sigma_{\bar{x}_{n}} .
\end{aligned}
$$

In the classical Kalman filter theory (e.g. Brown and Hwang), Eq. (56) is interpreted as correction of the state prediction. The Kalman gain is determined such that the trace of the covariance matrix of the state update is minimized, i.e.

$$
\begin{aligned}
& \hat{K}_{n}=\arg \min _{K_{n}}\left(\operatorname{trace}\left(\Sigma_{\hat{x}_{n}^{+}}\left(K_{n}\right)\right)\right) \\
& =\Sigma_{\bar{x}_{n}} H_{n}^{\mathrm{T}}\left(H_{n} \Sigma_{\bar{x}_{n}} H_{n}^{\mathrm{T}}+\Sigma_{z_{n}}\right)^{-1},
\end{aligned}
$$

which is consistent with Eq. (54).

We initialize the Kalman filter by a least-squares estimation of the baseline, differential tropospheric zenith delay and ambiguities, i.e. without an estimation of pseudorange multipath errors to overcome the rank defect.

\section{B. Ambiguity Fixing}

In this subsection, we describe the fixing of the floatvalued ambiguity estimates to integer numbers. Integer candidate vectors are determined with the famous Least-Squares AMBiguity Decorrelation Adjustment (LAMBDA) method of Teunissen [12]. The LAMBDA method performs a search of all integer candidate vectors $N_{u r}^{j}$ inside a pre-defined search space volume $\chi^{2}$, i.e.

$$
\left\|\hat{N}_{u r}-N_{u r}\right\|_{\Sigma_{\hat{N} u r}^{-1}}^{2} \stackrel{!}{\leq} \chi^{2}
$$

where $\hat{N}_{u r}$ denotes the float ambiguity estimates of the Kalman filter.

The search space volume $\chi^{2}$ is obtained from the volume of the unit sphere and the float ambiguity covariance as described in details in the well-known paper of Teunissen et al. [24].

A candidate selection based on the sum of squared ambiguity residuals is not sufficiently trustworthy due to eventual biases in the float ambiguities and/ or their covariance matrix. Henkel and Günther [25] proposed a partial integer decorrelation to reduce the amplification of biases by the decorrelation transformation. Nevertheless, phase multipath, azimuthal inhomogeneities of the troposphere, and unavoidable errors in the float ambiguity covariance matrix need an advanced integer selection technique.

We consider phase-only measurements for the candidate selection (to be independent from code multipath), and model the phase measurements according to Eq. (19) and (7) at epoch $n$ by

$$
\lambda \tilde{\varphi}_{u r, n}=H_{n}^{\mathrm{red}}\left(\begin{array}{c}
\vec{x}_{u r, n} \\
T_{\mathrm{z}, u r, n}
\end{array}\right)+\lambda N_{u r}+\lambda \Delta \varphi_{\mathrm{MP}, u r, n}+\varepsilon_{\lambda \varphi_{u r, n}}
$$

with the reduced mapping matrix

$$
H_{n}^{\mathrm{red}}=\left(\begin{array}{c|c|c}
\left(\vec{e}_{u}^{1 l}\right)^{\mathrm{T}} & m_{r}^{1 l} \\
\vdots & \vdots \\
\left(\vec{e}_{u}^{K l}\right)^{\mathrm{T}} & m_{r}^{K l}
\end{array}\right),
$$

and the phase noise

$$
\varepsilon_{\lambda \varphi_{u r, n}} \sim \mathcal{N}\left(0, \Sigma_{\lambda \varphi}\right)
$$

with covariance matrix $\Sigma_{\lambda \varphi}$. The baseline and tropospheric zenith delay are estimated from the phase-only measurements of Eq. (60) for each candidate vector $\check{N}_{u r}^{j}$ and epoch $n$ by least-squares estimation, i.e.

$$
\begin{aligned}
& \left(\begin{array}{c}
\check{\breve{x}}_{u r, n}\left(\check{N}_{u r}^{j}\right) \\
\check{T}_{\mathrm{z}, u r, n}\left(\check{N}_{u r}^{j}\right)
\end{array}\right) \\
& =\min _{\vec{x}_{u r, n}, T_{z, u r, n}}\left\|\lambda\left(\tilde{\varphi}_{u r, n}-\check{N}_{u r}^{j}\right)-H_{n}^{\mathrm{red}}\left(\begin{array}{c}
\vec{x}_{u r, n} \\
T_{\mathrm{z}, u r, n}
\end{array}\right)\right\|_{\Sigma_{\lambda \varphi}^{-1}}^{2} .
\end{aligned}
$$

The sum of squared measurement residuals of Eq. (63) is further developed as

$$
\begin{aligned}
\left\|\lambda\left(\tilde{\varphi}_{u r, n}-\check{N}_{u r}^{j}\right)-H_{n}^{\mathrm{red}}\left(\begin{array}{c}
\vec{x}_{u r, n} \\
T_{\mathrm{z}, u r, n}
\end{array}\right)\right\|_{\Sigma_{\lambda \varphi}^{-1}}^{2} \\
=\lambda\left(\tilde{\varphi}_{u r, n}-\check{N}_{u r}^{j}\right)^{\mathrm{T}} \Sigma_{\lambda \varphi}^{-1} \lambda\left(\tilde{\varphi}_{u r, n}-\check{N}_{u r}^{j}\right) \\
\quad-2\left(\begin{array}{c}
\vec{x}_{u r, n} \\
T_{\mathrm{z}, u r, n}
\end{array}\right)^{\mathrm{T}}\left(H_{n}^{\mathrm{red}}\right)^{\mathrm{T}} \Sigma_{\lambda \varphi}^{-1} \lambda\left(\tilde{\varphi}_{u r, n}-\check{N}_{u r}^{j}\right) \\
\quad+\left(\begin{array}{c}
\vec{x}_{u r, n} \\
T_{\mathrm{z}, u r, n}
\end{array}\right)^{\mathrm{T}}\left(H_{n}^{\mathrm{red}}\right)^{\mathrm{T}} \Sigma_{\lambda \varphi}^{-1}\left(H_{n}^{\mathrm{red}}\right)\left(\begin{array}{c}
\vec{x}_{u r, n} \\
T_{\mathrm{z}, u r, n}
\end{array}\right) .
\end{aligned}
$$


This sum of squared residuals is minimized by setting the derivative to zero, i.e.

$$
\begin{gathered}
\frac{\partial}{\partial\left(\begin{array}{c}
\vec{x}_{u r, n} \\
T_{\mathrm{z}, u r, n}
\end{array}\right)}\left\|\lambda\left(\tilde{\varphi}_{u r, n}-\check{N}_{u r}^{j}\right)-H_{n}^{\mathrm{red}}\left(\begin{array}{c}
\vec{x}_{u r, n} \\
T_{\mathrm{z}, u r, n}
\end{array}\right)\right\|_{\Sigma_{\lambda \varphi}^{-1}}^{2} \\
=-2\left(H_{n}^{\mathrm{red}}\right)^{\mathrm{T}} \Sigma_{\lambda \varphi}^{-1} \lambda\left(\tilde{\varphi}_{u r, n}-\check{N}_{u r}^{j}\right) \\
\quad+2\left(H_{n}^{\mathrm{red}}\right)^{\mathrm{T}} \Sigma_{\lambda \varphi}^{-1}\left(H_{n}^{\mathrm{red}}\right)\left(\begin{array}{c}
\vec{x}_{u r, n} \\
T_{\mathrm{z}, u r, n}
\end{array}\right) \stackrel{!}{=} 0 .
\end{gathered}
$$

Solving the last equation for the unknown position and tropospheric parameters gives the least-squares solution:

$$
\left(\begin{array}{c}
\check{\vec{x}}_{u r, n}\left(\check{N}_{u r}^{j}\right) \\
\check{T}_{\mathrm{z}, u r, n}\left(\check{N}_{u r}^{j}\right)
\end{array}\right)=S_{n} \lambda\left(\tilde{\varphi}_{u r, n}-\check{N}_{u r}^{j}\right),
$$

with

$$
S_{n}=\left(\left(H_{n}^{\mathrm{red}}\right)^{\mathrm{T}} \Sigma_{\lambda \varphi}^{-1} H_{n}^{\mathrm{red}}\right)^{-1}\left(H_{n}^{\mathrm{red}}\right)^{\mathrm{T}} \Sigma_{\lambda \varphi}^{-1} .
$$

Replacing $\lambda \tilde{\varphi}_{u r, n}$ by Eq. (60) in Eq. (63) yields

$$
\begin{aligned}
& \left(\begin{array}{c}
\breve{\vec{x}}_{u r, n}\left(\check{N}_{u r}^{j}\right) \\
\check{T}_{\mathrm{z}, u r, n}\left(\check{N}_{u r}^{j}\right)
\end{array}\right)=\left(\begin{array}{c}
\vec{x}_{u r, n} \\
T_{\mathrm{z}, u r, n}
\end{array}\right) \\
& \quad+S_{n}\left(\lambda\left(N_{u r}-\check{N}_{u r}^{j}\right)+\lambda \Delta \varphi_{\mathrm{MP}, u r, n}+\varepsilon_{\lambda \varphi_{u r, n}}\right) .
\end{aligned}
$$

An ambiguity fixing error results in a drift of the baseline estimate as the $S_{n}$ matrix changes with the satellite geometry and, thus, with time. The true baseline is constant, i.e. any drift in the baseline estimate arises either from an ambiguity fixing error, phase multipath or phase noise, or any combination of these errors.

Therefore, we consider the time series of single epoch fixed baseline and tropospheric zenith delay estimates given by

$$
\begin{aligned}
\left(\begin{array}{c}
\check{\vec{x}}_{u r, 1}\left(\check{N}_{u r}^{j}\right) \\
\check{T}_{\mathrm{z}, u r, 1}\left(\check{N}_{u r}^{j}\right) \\
\vdots \\
\check{\vec{x}}_{u r, n}\left(\check{N}_{u r}^{j}\right) \\
\check{T}_{\mathrm{z}, u r, n}\left(\check{N}_{u r}^{j}\right)
\end{array}\right)= & \left(\begin{array}{cc}
I^{4 \times 4} & S_{1} \\
\vdots & \vdots \\
I^{4 \times 4} & S_{n}
\end{array}\right)\left(\begin{array}{c}
\vec{x}_{u r} \\
T_{\mathrm{z}, u r} \\
\lambda\left(N_{u r}-\check{N}_{u r}^{j}\right)
\end{array}\right) \\
& +\left(\begin{array}{c}
S_{1}\left(\lambda \Delta \varphi_{\mathrm{MP}, u r, 1}+\varepsilon_{\lambda \varphi_{u r, 1}}\right) \\
\vdots \\
S_{n}\left(\lambda \Delta \varphi_{\mathrm{MP}, u r, n}+\varepsilon_{\lambda \varphi_{u r, n}}\right)
\end{array}\right),
\end{aligned}
$$

with $I^{4 \times 4}$ being the identity matrix with 4 rows and columns. The $S_{n}$ matrix is changing only slowly with the satellite geometry, and is therefore approximated by

$$
S_{n} \approx S_{1}+\dot{S} \cdot\left(t_{n}-t_{1}\right) \text {. }
$$

We subtract the baseline and tropospheric zenith delay estimates of the first epoch from Eq. (69) and use Eq. (70) to obtain the change of the baseline and tropospheric zenith delay estimates over time:

$$
\begin{aligned}
& \left(\begin{array}{c}
\breve{\vec{x}}_{u r, 2}\left(\check{N}_{u r}^{j}\right)-\breve{\breve{x}}_{u r, 1}\left(\check{N}_{u r}^{j}\right) \\
\check{T}_{\mathrm{z}, u r, 2}\left(\check{N}_{u r}^{j}\right)-\breve{T}_{\mathrm{z}, u r, 1}\left(\check{N}_{u r}^{j}\right) \\
\vdots \\
\check{\vec{x}}_{u r, n}\left(\check{N}_{u r}^{j}\right)-\check{\vec{x}}_{u r, 1}\left(\check{N}_{u r}^{j}\right) \\
\check{T}_{\mathrm{T}, u r, n}\left(\check{N}_{u r}^{j}\right)-\check{\breve{T}}_{\mathrm{z}, u r, 1}\left(\check{N}_{u r}^{j}\right)
\end{array}\right) \\
& \approx\left(\left(\begin{array}{c}
t_{2}-t_{1} \\
\vdots \\
t_{n}-t_{1}
\end{array}\right) \otimes I^{4 \times 4}\right) \dot{S} \cdot\left(\lambda\left(N_{u r}-\check{N}_{u r}^{j}\right)\right)+e_{u r}
\end{aligned}
$$

with $e_{u r}$ being the combined phase multipath and noise given by

$$
\begin{aligned}
& e_{u r}=\left(\begin{array}{c}
S_{2}\left(\lambda \Delta \varphi_{\mathrm{MP}, u r, 2}+\varepsilon_{\lambda \varphi_{u r, 2}}\right) \\
\vdots \\
S_{n}\left(\lambda \Delta \varphi_{\mathrm{MP}, u r, n}+\varepsilon_{\lambda \varphi_{u r, n}}\right)
\end{array}\right) \\
& -\left(\begin{array}{c}
S_{1}\left(\lambda \Delta \varphi_{\mathrm{MP}, u r, 1}+\varepsilon \lambda \varphi_{u r, 1}\right) \\
\vdots \\
S_{1}\left(\lambda \Delta \varphi_{\mathrm{MP}, u r, 1}+\varepsilon_{\lambda \varphi_{u r, 1}}\right)
\end{array}\right),
\end{aligned}
$$

with $\otimes$ being the Kronecker operator. The phase multipath and phase noise are assumed to be Gaussian distributed. Thus, the slope of the baseline and tropospheric drifts due to an erroneous fixing is obtained from Eq. (71) by taking the expectation value over the noise and phase multipath and by normalizing with $t_{n}-t_{1}$, i.e.

$$
\begin{aligned}
& \mathrm{E}\left\{\frac{1}{t_{n}-t_{1}}\left(\begin{array}{c}
\check{\breve{x}}_{u r, n}\left(\check{N}_{u r}^{j}\right)-\check{\breve{x}}_{u r, 1}\left(\check{N}_{u r}^{j}\right) \\
\check{T}_{\mathrm{z}, u r, n}\left(\check{N}_{u r}^{j}\right)-\check{T}_{\mathrm{z}, u r, 1}\left(\check{N}_{u r}^{j}\right)
\end{array}\right)\right\} \\
& =\dot{S}\left(\lambda\left(N_{u r}-\check{N}_{u r}^{j}\right) .\right.
\end{aligned}
$$

Obviously, the slope is proportional to the ambiguity fixing error $N_{u r}-\breve{N}_{u r}^{j}$ and is 0 for a correct fixing. Therefore, we choose the ambiguity candidate that minimizes the squared norm of the baseline and tropospheric drifts, i.e.

$$
j_{\text {opt }}=\arg \min _{j} \sum_{i=2}^{n}\left\|\frac{1}{t_{i}-t_{1}}\left(\begin{array}{c}
\check{\vec{x}}_{u r, i}\left(\check{N}_{u r}^{j}\right)-\check{\vec{x}}_{u r, 1}\left(\check{N}_{u r}^{j}\right) \\
\check{T}_{\mathrm{z}, u r, i}\left(\check{N}_{u r}^{j}\right)-\breve{T}_{\mathrm{z}, u r, 1}\left(\check{N}_{u r}^{j}\right)
\end{array}\right)\right\|^{2} .
$$

The magnitude of the baseline/ tropospheric drift has to be larger than the combined phase multipath and noise to enable a reliable candidate selection. The drift increases linear with time. Therefore, a minimum number of epochs $n$ is needed.

The residuals of the fixed phase solution are obtained from Eq. (60) and (74):

$$
r_{u r}:=P_{H_{n}^{\text {red }}}^{\perp}\left(\lambda\left(\tilde{\varphi}_{u r, n}-\check{N}_{u r}^{j_{\text {opt }}}\right)\right),
$$

where the projector on the space orthogonal of $H_{n}^{\text {red }}$ is given by

$$
P_{H_{n}^{\text {red }}}^{\perp}=1-H_{n}^{\mathrm{red}}\left(\left(H_{n}^{\mathrm{red}}\right)^{\mathrm{T}} \Sigma_{\lambda \varphi}^{-1} H_{n}^{\mathrm{red}}\right)^{-1}\left(H_{n}^{\mathrm{red}}\right)^{\mathrm{T}} \Sigma_{\lambda \varphi}^{-1} .
$$

The ambiguity fixing is triggered once the baseline estimates of the Kalman filter are sufficiently stable over time. This stability-oriented criterion takes both measurement and 


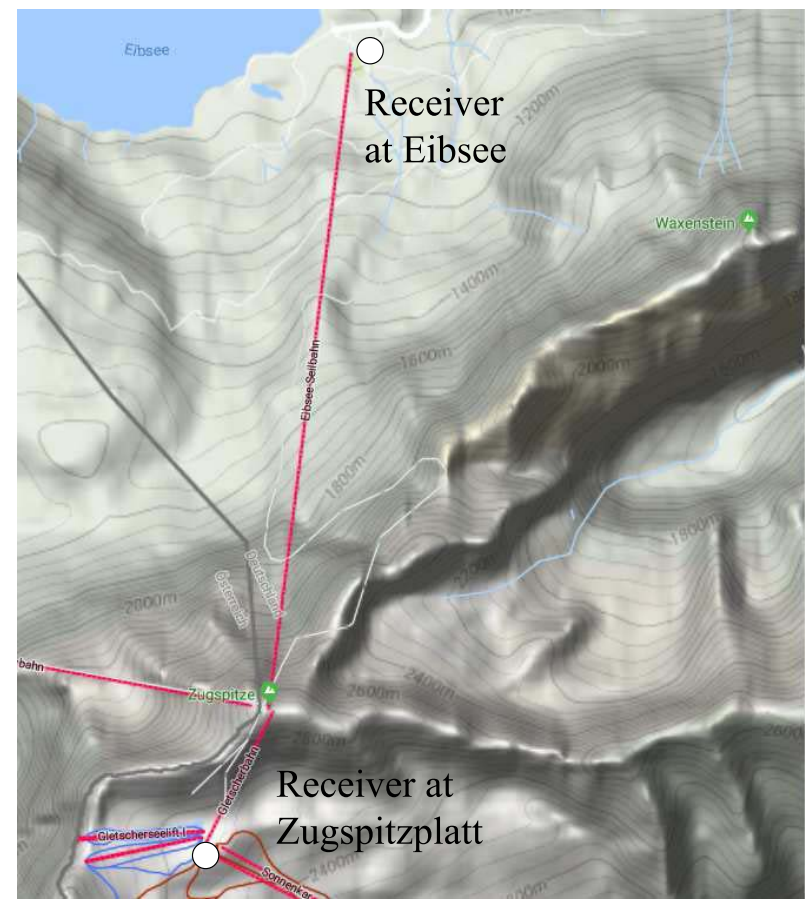

Fig. 2. Map with locations of ANavS GNSS receiver modules at Eibsee and Zugspitzplatt.

modeling errors into account and, therefore, is much more robust to these errors than the traditionally used covariance matrix of the Kalman filter's state update which does not depend on the actual measurements.

\section{Measurement Results}

In this section, the RTK positioning with differential tropospheric zenith delay and pseudorange multipath estimation is validated with real measurements.

\section{A. Measurement Set-Up}

The validation of the differential tropospheric zenith delay estimation requires a substantial height difference between both GNSS receivers. We have installed one ANavS GNSS receiver module at Zugspitzplatt (2601 $\mathrm{m}$ a.s.l.) and a second ANavS GNSS receiver module at Eibsee (1018 m a.s.1.) on Oct. 28, 2017. Fig. 2 shows the local topography and Fig. 3 includes a photo of the location of the environment of Zugspitzplatt. The GNSS receiver modules are equipped with the single-frequency Multi-GNSS receiver chip LEAM8T of u-blox, which provides pseudorange and carrier phase measurements with a rate of $5 \mathrm{~Hz}$.

The prior information on the differential tropospheric zenith delay is computed from Eq. (12) - (17) using a temperature lapse rate of $6.5 \mathrm{~K} / \mathrm{km}$, a reference height $h_{0}=0 \mathrm{~m}$, a temperature $T_{0}\left(h_{0}\right)=15^{\circ} \mathrm{C}$ and a total pressure $p\left(h_{0}\right)=1025 \mathrm{mbar}$. We obtained $36.7 \mathrm{~cm}$ for the hydrostatic component and approximated the wet component by $2 \mathrm{~cm}$ yielding a total differential zenith delay of $38.7 \mathrm{~cm}$.

\section{B. Kalman Filter Based Float Solution}

In this section, the performance of the Kalman filter based estimation of the baseline, differential tropospheric zenith

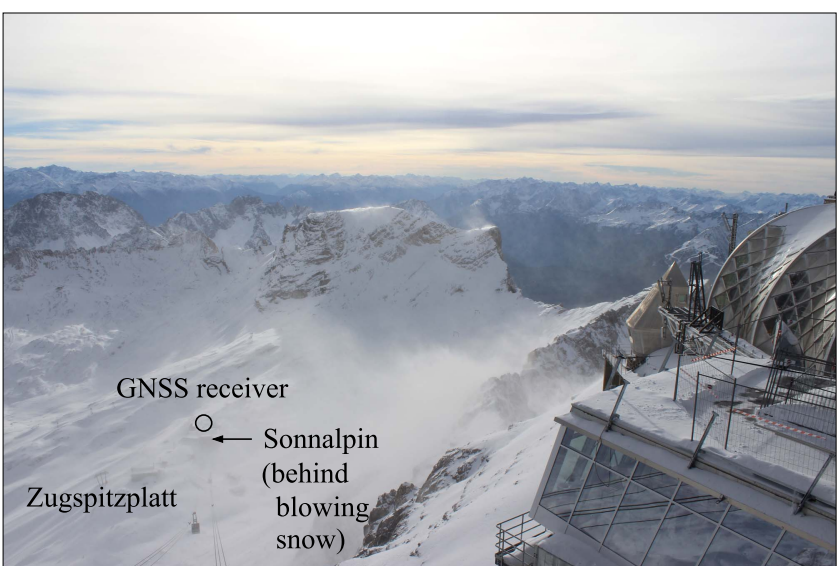

Fig. 3. Location of GNSS receiver at Zugspitzplatt.

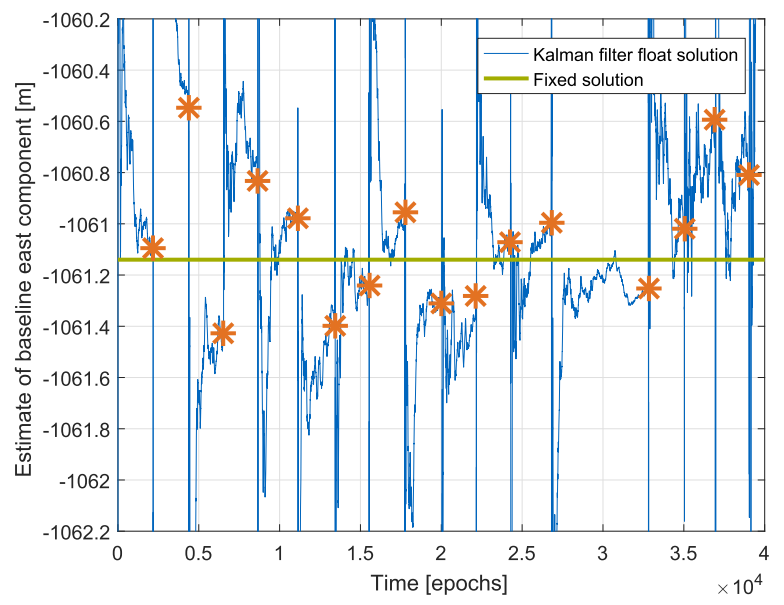

Fig. 4. Kalman filter based estimation of baseline east component: The *-symbols show the epochs of ambiguity fixings and (re-)initialization of the Kalman filter. A clear convergence can be observed after each (re-) initialization. The error of the baseline east component is below $0.5 \mathrm{~m}$ at the epoch of fixing.

delay, ambiguities and pseudorange multipath is analyzed. A separation of baseline, tropospheric zenith delay and ambiguities is enabled only by temporal changes of the satellitereceiver line of sight vectors and tropospheric mapping functions. This requires a minimum time span of measurements to be robust over phase multipath. The prior information on the troposphere helps to improve the conditioning of observation equations. Thereby, the correlation between the estimates of the differential tropospheric zenith delay and the baseline height component is reduced.

Fig. 4 and 5 show the performance of the baseline float solution. The ambiguity-fixed baseline serves as reference. The Kalman filter is (re-)initialized after each ambiguity fixing and the respective epochs are marked by a $*$-symbol. The baseline error converges to less than $0.5 \mathrm{~m}$ for the east and to less than $1.0 \mathrm{~m}$ for the up component. The prior knowledge of the differential tropospheric delay $\left(\sigma_{\bar{T}_{z, u r}}=5 \mathrm{~cm}\right)$ is important to constrain the uncertainty of the baseline up-component.

Fig. 6 describes the estimation of the pseudorange multipath errors during the first 2000 epochs. Both the estimates of the 


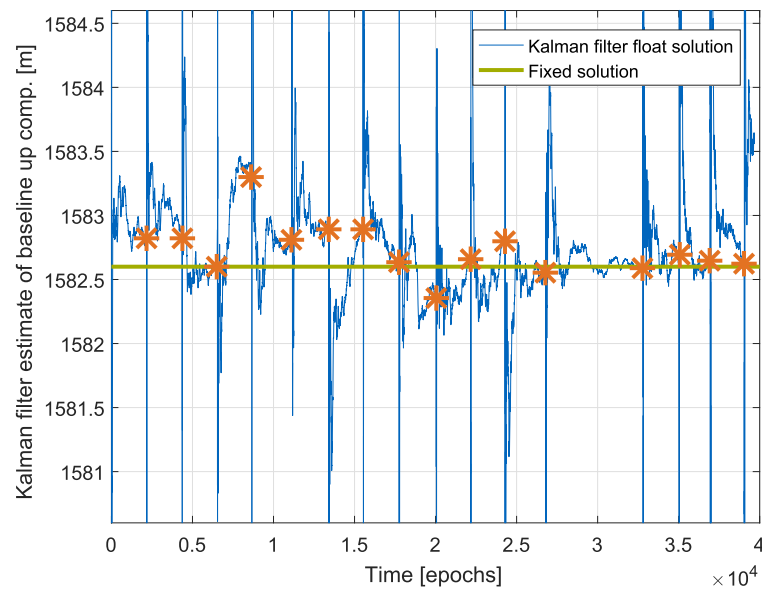

Fig. 5. Kalman filter based estimation of baseline up component: The *symbols show again the epochs of ambiguity fixings and (re-)initialization of the Kalman filter. The error of the baseline up component is below $1 \mathrm{~m}$ at the epoch of fixing.

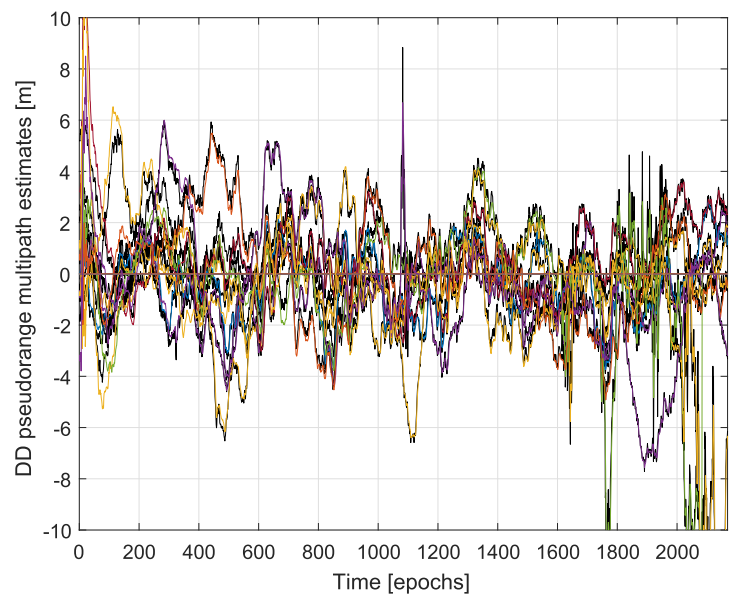

Fig. 6. Kalman filter based estimation of pseudorange multipath errors: The estimates of the pseudorange multipath errors are shown for different satellites in different colors. The short- to midterm-variations of the pseudorange multipath estimates are highly correlated with the short- to midterm-variations of the pseudorange measurements, which are also shown in black.

pseudorange multipath errors (one color for each satellite) and the double difference pseudorange measurements (in black) are shown.

The latter ones were corrected for the baseline and tropospheric zenith delay of the fixed solution to enable a direct comparison. We can observe that the estimates of the DD pseudorange multipath are extremely close to the pseudorange measurements after an initial convergence of $\sim 300$ epochs. The accurate estimation of pseudorange multipath errors is a very important result since low-cost patch antennas can hardly suppress multipath. Moreover, we exploit the temporal correlation of multipath and prevent a projection of multipath errors into other states.

\section{Fixed Solution}

In this sub-section, we describe the performance of the fixed baseline and differential tropospheric zenith delay estimates.

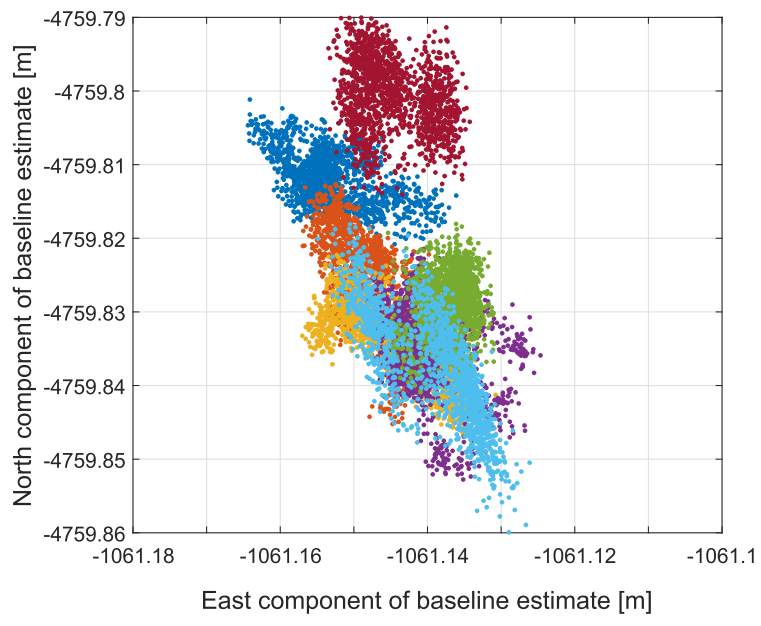

(a)

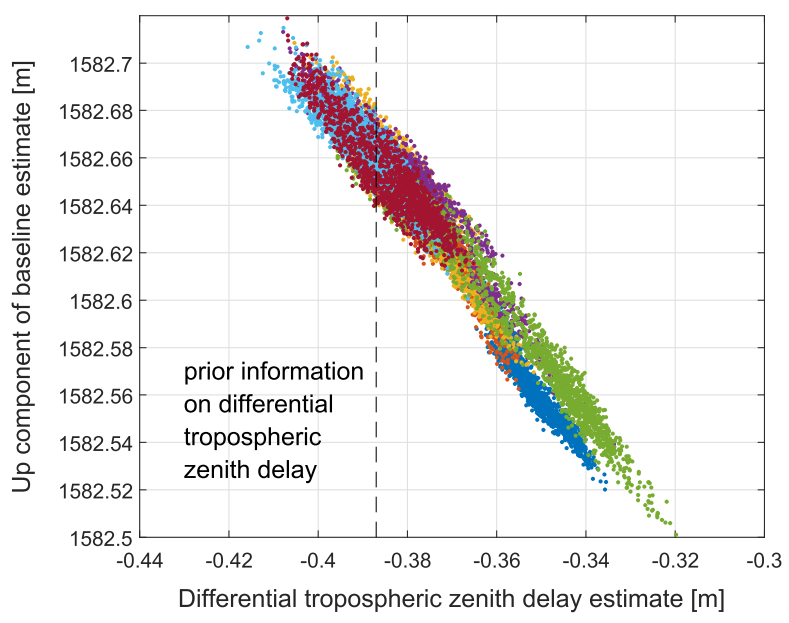

(b)

Fig. 7. Baseline estimates between Eibsee and Zugspitzplatt for 7 independent ambiguity fixings: The point cloud of each color refers to a separate dataset with a period of 5 minutes. The slight movement of the point clouds is caused primarily by phase multipath and changes in the satellite geometry. (a) Baseline east versus north component: The local topography at Eibsee blocks the signals of all satellites from southern direction with an elevation of less than 30 degrees. Therefore, the north component shows a larger variation than the east component. (b) Baseline up component versus tropospheric delay: A high negative correlation can be observed between the estimates of the baseline up component and the differential tropospheric zenith delay.

Fig. 7a shows the baseline east and north components for 7 independent ambiguity fixings.

All point clouds are within a few centimeters, which indicates correct integer fixings. We can also observe that the variation in the North component is roughly twice as large as the variation in the East component. This is a consequence of the local topography at Eibsee (see Fig. 2) leading to a substantially reduced visibility of satellites from Southern direction. The small offsets between the point clouds are caused by phase multipath and changes of the satellite geometry (e.g. lost/ new satellites).

Fig. 7b shows the baseline up-component as a function of the differential tropospheric zenith delay estimate. Each point cloud is referring again to a separate ambiguity 


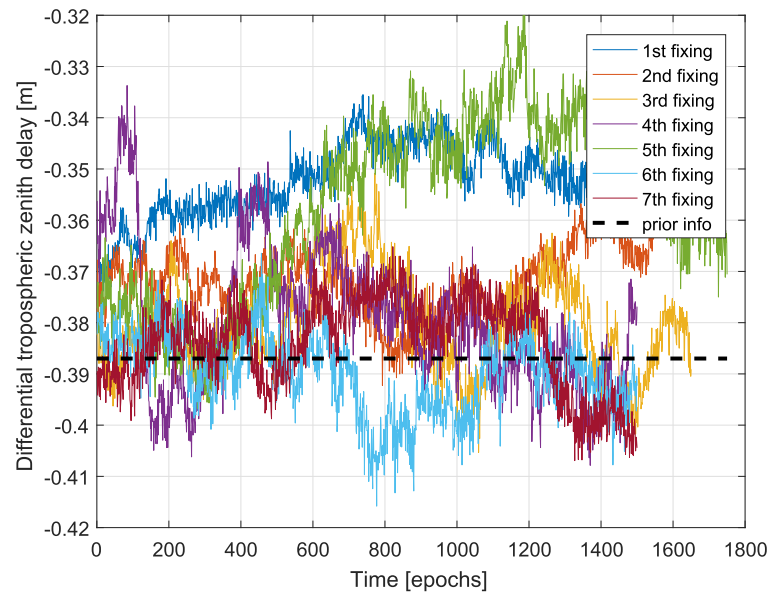

Fig. 8. Differential tropospheric zenith delay estimates over time for 7 independent ambiguity fixings.

fixing. The overlapping point clouds and their small offsets of only a few centimeters indicate again a correct integer fixing. We can also observe a very high negative correlation between the estimates of the baseline up component and the differential tropospheric zenith delay. A baseline height error $\Delta h$ propagates into the range error $\Delta r^{k}=\sin \left(E^{k}\right) \Delta h$ and can thus partially compensate for a tropospheric range error $T_{z, u r} / \sin \left(E_{r}^{k}\right)$. The correlation is already reduced due to the integration of prior information on the troposphere and would otherwise be even significantly larger.

Fig. 8 shows the differential tropospheric zenith delay over time for the same ambiguity fixings. The self-consistency of the estimates, their consensus with the tropospheric prior information (shown as dashed line), and the lack of any significant drift indicate correct integer ambiguity fixings. The temporal variations of the tropospheric zenith delay estimates are primarily caused by phase multipath and noise, and only to a very small extent eventually by changes in the weather (humidity). The 1st and 5th fixing show a slight offset in the tropospheric zenith delay due to an increased phase multipath. This error also propagates into the baseline height being shown in Fig. 7b. The strong negative correlation between the estimated baseline height and differential tropospheric zenith delay implies that respective errors mostly cancel in the residuals.

Fig. 9 and 10 show the residuals of the fixed carrier phase measurements for the ambiguity fixings of Fig. 7. The figure also shows tropospheric residuals, i.e. the deviation between the prior information and the actual estimate of the differential tropospheric zenith delay estimate. The noise level of the tropospheric residuals is larger than for the phase residuals since the GNSS carrier phase measurements are much more accurate (with typical standard deviations between $2 \mathrm{~mm}$ and $1 \mathrm{~cm}$ ) and, therefore, weighted 5 to 25 times higher than the tropospheric prior information (standard deviation of $5 \mathrm{~cm}$ ).

The phase residuals are within $\pm 2 \mathrm{~cm}$ and without any longterm drift for most satellites, which indicates again correct integer fixings. We can also observe that two or three satellites show a slightly larger noise level and small biases or drifts.

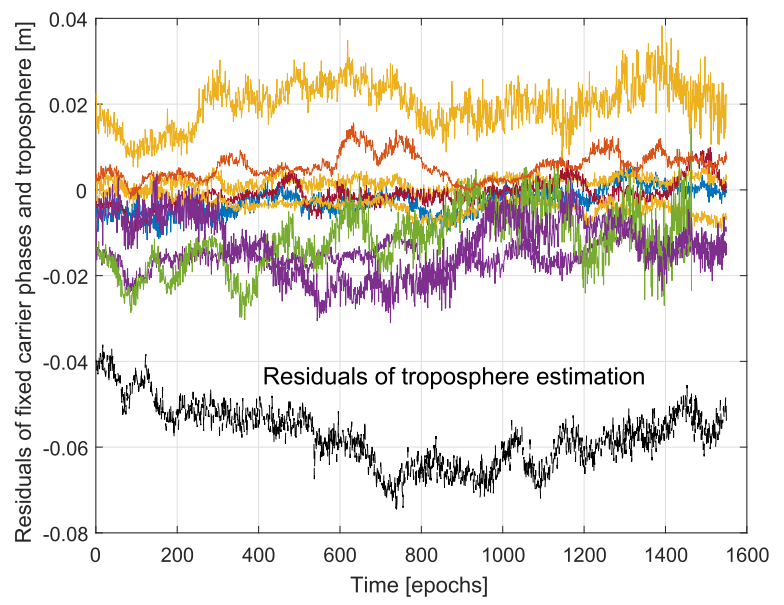

(a)

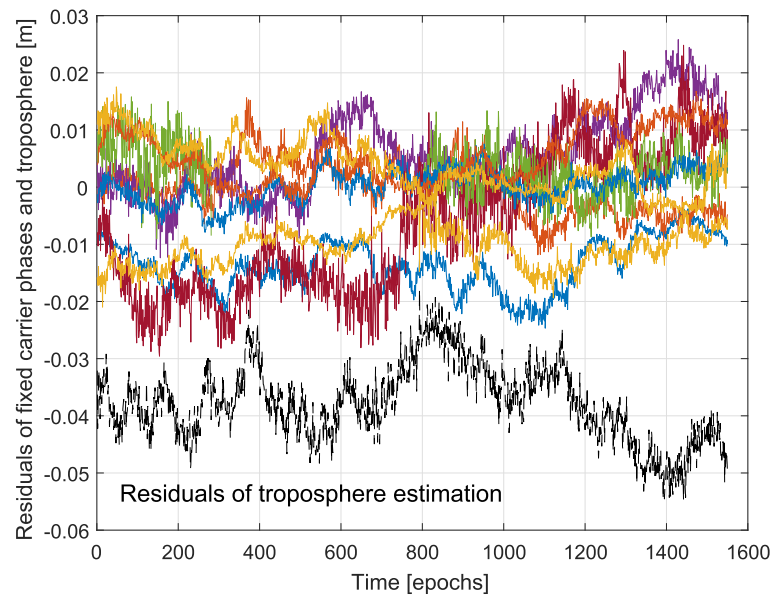

(b)

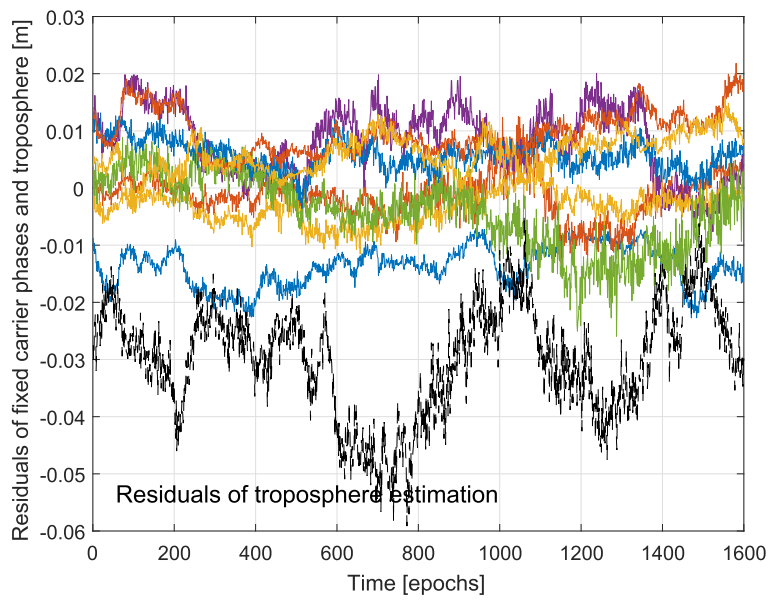

(c)

Fig. 9. Residuals of fixed carrier phase measurements and tropospheric delay information for first three ambiguity fixings. Different colors refer to different satellites. (a) First ambiguity fixing (b) 2nd ambiguity fixing (c) 3rd ambiguity fixing.

These satellites have a low elevation leading to increased phase multipath and a larger impact of inhomogeneities in the troposphere. In Fig. 10c, these satellites are set leading to residual errors of less than $1 \mathrm{~cm}$. 


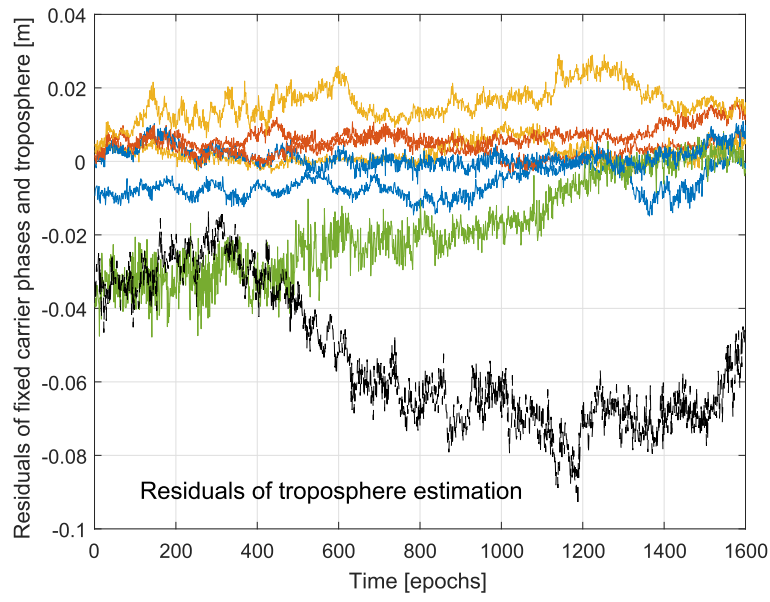

(a)

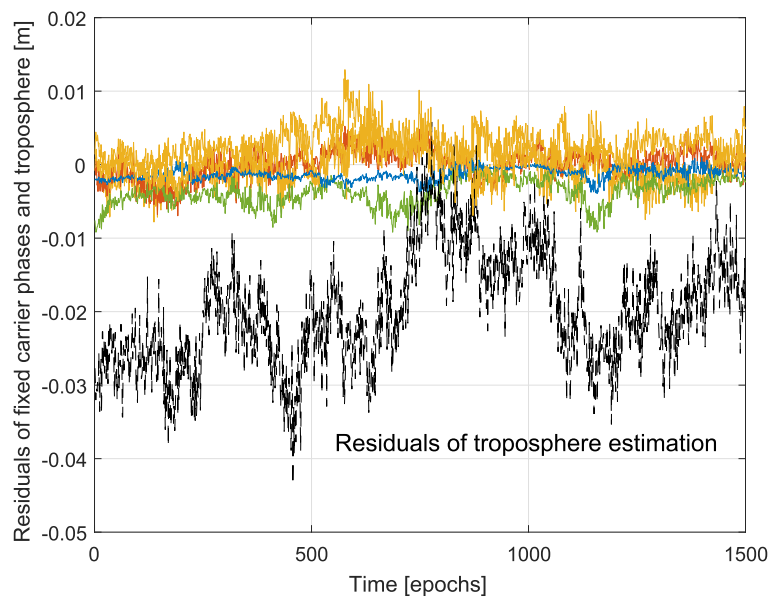

(b)

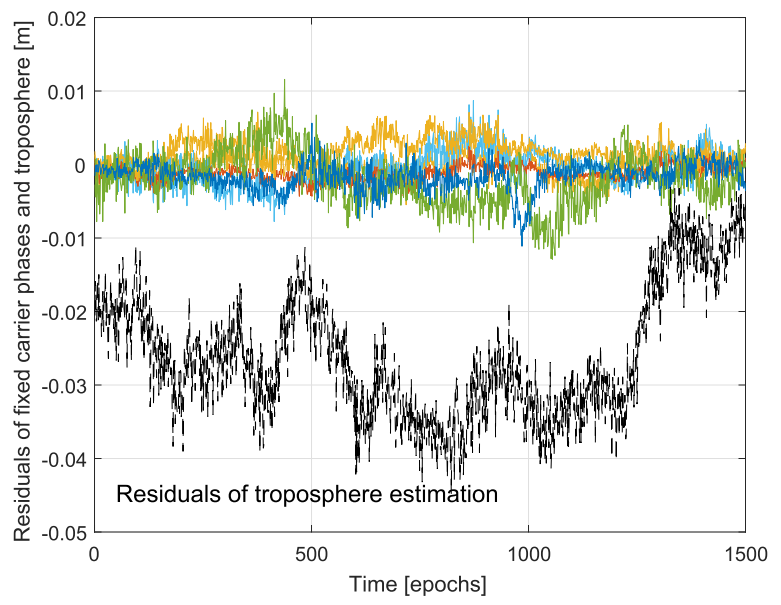

(c)

Fig. 10. Residuals of fixed carrier phase measurements and tropospheric delay information for subsequent ambiguity fixings. Different colors refer to different satellites. (a) 5th ambiguity fixing (b) 6th ambiguity fixing (c) 7 th ambiguity fixing.

\section{CONClusion}

The reliability of Real-Time Kinematic (RTK) positioning in Alpine areas with low-cost GNSS receivers and patch antennas is degraded by pseudorange and carrier phase multipath.
Moreover, the differential tropospheric delays are no longer negligible for height differences of more than $100 \mathrm{~m}$.

In this paper, an extended RTK positioning was introduced with a joint estimation of the baseline, differential tropospheric zenith delay, carrier phase integer ambiguities and pseudorange multipath errors. Moreover, a criterion for robust integer ambiguity candidate selection was suggested, that is robust over phase multipath and unavoidable errors in the float ambiguity covariance matrix. The extended RTK positioning was tested at Germany's highest mountain - the Zugspitze. The measurement results showed a high baseline repeatability over different ambiguity fixings. A single-epoch positioning accuracy of a few centimeters was achieved after ambiguity fixing. The accuracy could be further improved by filtering of the position estimates.

The extended RTK positioning for low-cost GNSS receivers and antennas seems to be well-suited for surveying, landslide monitoring and precise positioning of autonomous ground or aerial vehicles.

\section{REFERENCES}

[1] M. Sahmoudi and R. Landry, "A nonlinear filtering approach for robust multi-GNSS RTK positioning in presence of multipath and ionospheric delays," IEEE J. Sel. Topics Signal Process., vol. 3, no. 5, pp. 764-776, Oct. 2009

[2] T. Takasu and A. Yasuda, "Kalman-filter-based integer ambiguity resolution strategy for long-baseline RTK with ionosphere and troposphere estimation," in Proc. ION GNSS, Portland, OR, USA, Sep. 2010, pp. 161-171.

[3] H. Wymeersch, J. Lien, and M. Z. Win, "Cooperative localization in wireless networks," Proc. IEEE, vol. 97, no. 2, pp. 427-450, Feb. 2009.

[4] H. M. Georges, Z. Xiao, and D. Wang, "Hybrid cooperative vehicle positioning using distributed randomized sigma point belief propagation on non-Gaussian noise distribution," IEEE Sensors J., vol. 16, no. 21, pp. 7803-7813, Nov. 2016.

[5] N. Alam and A. G. Dempster, "Cooperative positioning for vehicular networks: Facts and future," IEEE Trans. Intell. Transp. Syst., vol. 14, no. 4, pp. 1708-1717, Dec. 2013.

[6] S. Zhao, Y. Chen, and J. A. Farrell, "High-precision vehicle navigation in urban environments using an MEM's IMU and single-frequency GPS receiver," IEEE Trans. Intell. Transp. Syst., vol. 17, no. 10, pp. 2854-2867, Oct. 2016.

[7] P. S. Henkel, "Tightly coupled precise point positioning and attitude determination," IEEE Trans. Aerosp. Electron. Syst., vol. 51, no. 4, pp. 3182-3197, Oct. 2015.

[8] G. Elgered, J. L. Davis, T. A. Herring, and I. I. Shapiro, "Geodesy by radio interferometry: Water vapor radiometry for estimation of the wet delay," J. Geophys. Res., vol. 96, no. B4, pp. 6541-6555, 1991.

[9] M. Bevis, S. Businger, T. A. Herring, C. Rocken, R. A. Anthes, and R. H. Ware, "GPS meteorology: Remote sensing of atmospheric water vapor using the global positioning system," J. Geophys. Res., vol. 97, no. D14, pp. 15787-15801, Oct. 1992, doi: 10.1029/92JD01517.

[10] Y. W. Ahn, D. Kim, and P. Dare, "Local tropospheric anomaly effects on GPS RTK performance," in Proc. 19th Int. Technol. Meeting ION, Fort Worth, TX, USA, Sep. 2006, pp. 1925-1935.

[11] H. Landau, U. Vollath, and X. Chen, "Virtual reference station systems," J. Global Positioning Syst., vol. 1, no. 2, pp. 137-143, 2002, doi: 10.5081/jgps.1.2.137.

[12] P. J. G. Teunissen, "The least-squares ambiguity decorrelation adjustment: A method for fast GPS integer ambiguity estimation," J. Geodesy, vol. 70, nos. 1-2, pp. 65-82, 1995, doi: 10.1007/BF00863419.

[13] P. Henkel, U. Mittmann, and M. Iafrancesco, "Real-time kinematic positioning with GPS and GLONASS," in Proc. 24th Eur. Signal Process. Conf. (EUSIPCO), Budapest, Hungary, Sep. 2016, pp. 1063-1067, doi: 10.1109/EUSIPCO.2016.7760411.

[14] P. Misra and P. Enge, Global Positioning System: Signals, Measurements, and Performance, 2nd ed. Lincoln, MA, USA: Ganga-Jamuna Press, 2011. 
[15] J. L. Davis, T. A. Herring, I. I. Shapiro, A. E. E. Rogers, and G. Elgered, "Geodesy by radio interferometry: Effects of atmospheric modeling errors on estimates of baseline length," Radio Sci., vol. 20, no. 6, pp. $1593-1607,1985$

[16] H. S. Hopfield, "Two-quartic tropospheric refractivity profile for correcting satellite data," J. Geophys. Res., vol. 74, no. 18, pp. 4487-4499, 1969.

[17] Y. Zhang and C. Bartone, "Single-site carrier phase based troposphere integrity monitoring," in Proc. Nat. Technol. Meeting ION, Monterey, CA, USA, Jan. 2006, pp. 530-542.

[18] P. Henkel, M. Iafrancesco, and A. Sperl, "Precise point positioning with multipath estimation," in Proc. ION/ IEEE Position, Location Navigat. Symp. (PLANS), Savannah, GA, USA, Apr. 2016, pp. 144-149, doi: 10.1109/PLANS.2016.7479694.

[19] R. G. Brown and P. Y. C. Hwang, Introduction to Random Signals and Applied Kalman Filtering With MATLAB Exercises, 4th ed. Hoboken, NJ, USA: Wiley, 2012.

[20] D. Bickson, "Gaussian belief propagation: Theory and application," Ph.D. dissertation, Hebrew Univ. Jerusalem, Jerusalem, Israel, Jul. 2009.

[21] Y. Weiss and W. Freeman, "Correctness of belief propagation in Gaussian graphical models of arbitrary topology," Neural Comput., vol. 13 , no. 10 , pp. $2173-2200$, Oct. 2001.

[22] J. Du, S. Ma, Y.-C. Wu, S. Kar, and J. M. F. Moura, "Convergence analysis of distributed inference with vector-valued Gaussian belief propagation," J. Mach. Learn. Res., vol. 38, no. 1, pp. 6302-6339, Dec. 2017.

[23] M. A. Woodbury, "Inverting modified matrices," Stat. Res. Group, Princeton Univ., Princeton, NJ, USA, Tech. Memo. 42, 1950, p. 4.
[24] P. J. G. Teunissen, P. J. de Jonge, and C. C. J. M. Tiberius, "The volume of the GPS ambiguity search space and its relevance for integer ambiguity resolution," in Proc. ION GNSS, Sep. 1996, pp. 889-898.

[25] P. Henkel and C. Günther, "Partial integer decorrelation: Optimum tradeoff between variance reduction and bias amplification," J. Geodesy, vol. 84, no. 1, pp. 51-63, 2010.

Patrick Henkel received the B.Sc., Dipl.Ing., and Ph.D. degrees from the Technical University of Munich, Munich, Germany, in 2004, 2005, and 2010, respectively. He was a Guest Researcher with TU Delft in 2007, Stanford University in 2008 and 2010, and Curtin University in 2013. He received a prestigious Award from the Vodafone Research Foundation for his Ph.D dissertation on reliable carrier phase positioning in 2010 and the Bavarian Regional Prize in the European Satellite Navigation Competition in 2011 for a differential carrier phase positioning method.

$\mathrm{He}$ is currently pursuing the Habilitation degree in precise point positioning. $\mathrm{He}$ is also one of the founders and managing directors of ANavS GmbH - Advanced Navigation Solutions.

Bigyan Banjara received the M.Sc. degree in earth oriented space science and technology (ESPACE) from the Technical University of Munich, Munich, Germany, in 2017. His master's thesis was on real-time kinematics with tropospheric delay estimation. He returned to his home country Nepal after his master studies. He is currently a Survey Officer with the Survey Department of the Government of Nepal. 